Noname manuscript No.

(will be inserted by the editor)

\title{
Dualities and Identities for Entanglement-Assisted Quantum Codes
}

\author{
Ching-Yi Lai · Todd A. Brun · Mark M. Wilde
}

Received: date / Accepted: date

\begin{abstract}
The dual of an entanglement-assisted quantum error-correcting (EAQEC) code is the code resulting from exchanging the original code's information qubits with its ebits. To introduce this notion, we show how entanglement-assisted (EA) repetition codes and accumulator codes are dual to each other, much like their classical counterparts, and we give an explicit, general quantum shift-register circuit that encodes both classes of codes. We later show that our constructions are optimal, and this result completes our understanding of these dual classes of codes. We also establish the Gilbert-Varshamov bound and the Plotkin bound for EAQEC codes, and we use these to examine the existence of some EAQEC codes. Finally, we provide upper bounds on the block error probability when transmitting maximal-entanglement EAQEC codes over the depolarizing channel, and we derive variations of the hashing bound for EAQEC codes, which is a lower bound on the maximum rate at which reliable communication over Pauli channels is possible with the use of pre-shared entanglement.
\end{abstract}

Keywords quantum dual code · entanglement-assisted quantum error correction · MacWilliams identity · linear programming bound · entanglement-assisted repetition codes · entanglement-assisted accumulator codes $\cdot$ hashing bound

PACS 03.67.-a 0 03.67.Pp

\section{Introduction}

The existence of quantum error correcting codes that have the ability to fight decoherence is one of the reasons why many believe that large-scale quantum computation and quantum communication will one day be possible [34, 37, 39, 13,2,19]. Quantum information can be protected against noise and decoherence by encoding it into quantum error-correcting codes, which introduce redundancy to the structure of quantum states.

Quantum stabilizer codes are an extensively analyzed class of quantum error-correcting codes, and they have many similarities with additive codes from classical error correction theory 66,7, 14, 26, In particular, a quantum code designer can produce quantum stabilizer codes from classical binary and quaternary self-orthogonal codes by means of the CSS and CRSS code constructions, respectively [8, 38, 6. 7.

Entanglement-assisted quantum error correction is a paradigm for quantum error correction in which a sender and receiver are allowed to share entanglement before quantum communication begins [5]. An $[[n, k, d ; c]]$ EAQEC code encodes $k$ information qubits into $n$ channel qubits with the help of $c$ pairs of maximally-entangled Bell states. The code can correct up to $\left\lfloor\frac{d-1}{2}\right\rfloor$ errors acting on the $n$ channel qubits,

Ching-Yi Lai and Todd A. Brun are with the Communication Sciences Institute, Electrical Engineering Department, University of Southern California, Los Angeles, California, USA 90089. E-mail: laiching@usc.edu and tbrun@usc.edu . Mark M. Wilde was with the School of Computer Science, McGill University, Montreal, Quebec, Canada H3A 2A7 when this research was conducted. He is now with the Hearne Institute for Theoretical Physics, the Department of Physics and Astronomy, and the Center for Computation and Technology at Louisiana State University, Baton Rouge, Louisiana 70803, USA E-mail: mwilde@gmail.com. 
where $d$ is the minimum distance of the code. Standard stabilizer codes are a special case of EAQEC codes with $c=0$, and we use the notation $[[n, k, d]]$ for such codes.

It has been shown that EAQEC codes have some advantages over standard stabilizer codes. For example, only a dual-containing classical linear quaternary code can be transformed into a standard stabilizer code, but any classical linear quaternary code can be transformed into an EAQEC code. Also, EA quantum LDPC codes with girth greater than or equal to six can be constructed, and they have good performance [16,17. Properties of EAQEC codes and their applications can be found in Refs. 44, 45, $40,20,21$.

The MacWilliams identity for quantum codes connects the weight enumerator of a classical quaternary self-orthogonal code associated with the quantum code to the weight enumerator of its dual code 33, 28, 29, 1. This type of MacWilliams identity for quantum stabilizer codes can be directly obtained by applying the Poisson summation formula from the theory of orthogonal groups 22. However, the orthogonal group of a stabilizer group with respect to the symplectic inner product (which will be defined below) does not define another quantum stabilizer code. So this is not a duality between codes in the usual quantum case.

Lai et al. defined a duality between entanglement-assisted quantum error-correcting codes based on the theory of orthogonal groups 22. This duality is very similar to the classical notion of duality, because the orthogonal group of an EA quantum code also forms a nontrivial EA quantum code.

This paper builds on and extends the results presented in Ref. [22], giving a more detailed exposition and including additional related topics:

- We demonstrate the duality discussed above with the example of repetition and accumulator EAQEC codes, together with their encoding circuits. Lai and Brun recently constructed a family of EA repetition codes with parameters $[[n, 1, n ; n-1]]$ for $n$ odd [20. Herein we produce a family of $[[n, 1, n-1 ; n-1]]$ EA repetition codes for $n$ even, which we prove to be optimal 1 thus completing the family of EA repetition codes for any $n$.

- By exploiting the linear programming bound derived from the MacWilliams identity for EAQEC codes [22, we can now show that several code parameters proposed in Ref. 20] are optimal.

- We establish the Gilbert-Varshamov bound for EAQEC codes, proving the existence of EAQEC codes with certain parameter values.

- We also derive the quantum version of the Plotkin bound [24, which is tight for codes with small $k$ and maximal entanglement.

- The table of upper and lower bounds on the highest achievable minimum distance of any maximalentanglement EAQEC code for $n \leq 15$ in [22] is established by combining the linear programming bounds [22] with the existence of some EAQEC codes established in this paper.

- The weight enumerator of a classical code gives an upper bound on the block error probability when transmitting coded bits through a binary symmetric channel [30,25. Since maximal-entanglement EAQEC codes have many similarities with classical codes 20,40, we can find an upper bound on the block error probability when transmitting coded quantum information through the depolarizing channel, and this derivation is similar to the classical derivation [30. We also exploit this result to find an upper bound on the expected block error probability when decoding a random maximalentanglement EAQEC code.

- The hashing bound of a quantum channel is an achievable rate for reliable quantum communication 2, and it has a simple form for Pauli channels. We first review a simple proof of the hashing bound for stabilizer codes [35] and then derive variations of the hashing bound for EAQEC codes. The proof exploits the method of random stabilizer coding.

We organize this paper as follows. We first review the basics of EAQEC codes and give the definition of the dual of an EAQEC code, and we explain this notion with the example of the dual repetition and accumulator EAQEC codes. We follow the terminology and notations of EAQEC codes used in Ref. 20]. For details, we point the reader to Refs. 5, 20. We review and study the duality, the MacWilliams identity, and the linear programming bound for EAQEC codes in Section 3 . In Section 4 , we begin with the Gilbert-Varshamov bound for EAQEC codes. We then describe the construction of $[[n, 1, n-1 ; n-1]]$ EA repetition codes for $n$ even and prove other results about the existence of EAQEC codes, including

\footnotetext{
1 An $[[n, k, d ; c]]$ EAQEC code is optimal in the sense that $d$ is the highest achievable minimum distance for given parameters $n, k$, and $c$.
} 
the EA Plotkin bound. Section 5 details an upper bound on the block error probability under maximumlikelihood decoding, and Section 6 summarizes variations of hashing bounds for EAQEC codes over Pauli channels. The final section concludes with a summary and open questions for future research.

\section{Preliminaries}

We begin with some notation. The Pauli matrices

$$
I=\left[\begin{array}{ll}
1 & 0 \\
0 & 1
\end{array}\right], X=\left[\begin{array}{ll}
0 & 1 \\
1 & 0
\end{array}\right], Y=\left[\begin{array}{cc}
0 & -i \\
i & 0
\end{array}\right], Z=\left[\begin{array}{cc}
1 & 0 \\
0 & -1
\end{array}\right]
$$

form a basis for the space of linear operators which act on a two-dimensional single-qubit state space. Let

$$
\mathcal{G}_{n}=\left\{e M_{1} \otimes \cdots \otimes M_{n}: M_{j} \in\{I, X, Y, Z\}, e \in\{ \pm 1, \pm i\}\right\}
$$

be the $n$-fold Pauli group. Any element $g=e M_{1} \otimes \cdots \otimes M_{n} \in \mathcal{G}_{n}$ can be expressed as $g=e^{\prime} X^{u} Z^{v}$, where $e^{\prime} \in\{ \pm 1, \pm i\} ; u=\left(u_{1} \cdots u_{n}\right)$ and $v=\left(v_{1} \cdots v_{n}\right)$ are two binary $n$-tuples defined as follows. If $M_{j}$ is $I, X, Z$, or $Y$, then $\left(u_{j}, v_{j}\right)=(0,0),(1,0),(0,1)$, or $(1,1)$, respectively. The weight wt $(g)$ of $g$ is the number of operators $M_{j}$ that are not equal to the identity operator $I$. We use the notation $X_{j}, Y_{j}$, or $Z_{j}$ to denote a Pauli operator which acts on qubit number $j$. Since the overall phase of a quantum state is not important, we consider the quotient of the Pauli group by its center $\overline{\mathcal{G}}_{n}=\mathcal{G}_{n} /\{ \pm 1, \pm i\}$, which is an Abelian group and can be generated by a set of $2 n$ independent generators. For $g_{1}=X^{u_{1}} Z^{v_{1}}$, $g_{2}=X^{u_{2}} Z^{v_{2}} \in \overline{\mathcal{G}}_{n}$, the symplectic inner product $*$ in $\overline{\mathcal{G}}_{n}$ is defined by

$$
g_{1} * g_{2}=u_{1} \cdot v_{2}+u_{2} \cdot v_{1} \bmod 2
$$

where is the usual inner product for binary $n$-tuples. Note that $*$ is commutative. We define a map $\phi$ : $\mathcal{G}_{n} \rightarrow \overline{\mathcal{G}}_{n}$ by $\phi\left(e X^{u} Z^{v}\right)=X^{u} Z^{v}$. For $g, h \in \mathcal{G}_{n}, \phi(g) * \phi(h)=0$ if $g$ and $h$ commute, and $\phi(g) * \phi(h)=1$, otherwise. The orthogonal group of a subgroup $V$ of $\overline{\mathcal{G}}_{n}$ with respect to $*$ is

$$
V^{\perp}=\left\{g \in \overline{\mathcal{G}}_{n}: g * h=0, \forall h \in V\right\} .
$$

For example, consider a stabilizer subgroup $\mathcal{S}$ of $\mathcal{G}_{n}$ and its normalizer group $N(\mathcal{S})$. Then the orthogonal group of $\phi(\mathcal{S})$ is $(\phi(\mathcal{S}))^{\perp}=\phi(N(\mathcal{S}))$.

An $[[n, k, d]]$ stabilizer code is a $2^{k}$-dimensional subspace of the $n$-qubit Hilbert space $\mathcal{H}^{\otimes n}$, and is the joint +1 -eigenspace of $n-k$ independent generators of a stabilizer subgroup $\mathcal{S}$ of $\overline{\mathcal{G}}_{n}$. The minimum distance $d$ is the minimum weight of any element in $\phi(N(\mathcal{S})) \backslash \phi(\mathcal{S})$.

We review some basics of EAQEC codes [5,20]. Suppose Alice and Bob share $c$ maximally-entangled pairs $\left|\Phi_{+}\right\rangle_{A B}=\frac{1}{\sqrt{2}}(|00\rangle+|11\rangle)$. Such a shared pair is called an ebit. Alice encodes a $k$-qubit state $|\phi\rangle$ by using an $[[n, k, d ; c]]$ EAQEC code with a Clifford encoder $U$, and then sends her $n$ qubits ( $k$ information qubits, $n-k-c$ ancilla qubits, and $c$ ebits) to Bob. A Clifford operator is a unitary operator that maps elements $\overline{\mathcal{G}}_{n}$ to elements of $\overline{\mathcal{G}}_{n}$ under unitary conjugation. Alice applies this encoder to her $k$ information qubits, $c$ shares of entangled pairs, and $n-k-c$ ancilla qubits prepared in the state $|0\rangle$, for a total of $n$ qubits on her side. We assume that Bob's qubits suffer no errors since they do not pass through the noisy channel. (The minimum distance $d$ will be defined later.) Suppose the initial state is $|\phi\rangle\left|\Phi_{+}\right\rangle_{A B}^{\otimes c}|0\rangle^{\otimes n-k-c}$. Let $g_{j}=U Z_{j} U^{\dagger}$ and $h_{j}=U X_{j} U^{\dagger}$ for $j=1, \cdots, n$ in $\overline{\mathcal{G}}_{n}$. The encoded state $U|\phi\rangle\left|\Phi_{+}\right\rangle_{A B}^{\otimes c}|0\rangle^{\otimes n-k-c}$ has a set of stabilizer generators

$$
\left\{g_{k+1}^{A} \otimes Z_{k+1}^{B}, \cdots, g_{k+c}^{A} \otimes Z_{k+c}^{B}, g_{k+c+1}^{A} \otimes I^{B}, \cdots, g_{n}^{A} \otimes I^{B}, h_{k+1}^{A} \otimes X_{k+1}^{B}, \cdots, h_{k+c}^{A} \otimes X_{k+c}^{B}\right\}
$$

in $\overline{\mathcal{G}}_{n+c}$, where the superscript $A$ or $B$ indicates that the operator acts on the qubits of Alice or Bob, respectively. The case where noise occurs on the ebits was considered in Refs. 31, 40,21.

The simplified stabilizer subgroup $\mathcal{S}^{\prime}$ of $\overline{\mathcal{G}}_{n}$ is

$$
\mathcal{S}^{\prime}=\left\langle g_{k+1}, \cdots, g_{k+c}, h_{k+1}, \cdots, h_{k+c}, g_{k+c+1}, \cdots, g_{n}\right\rangle .
$$




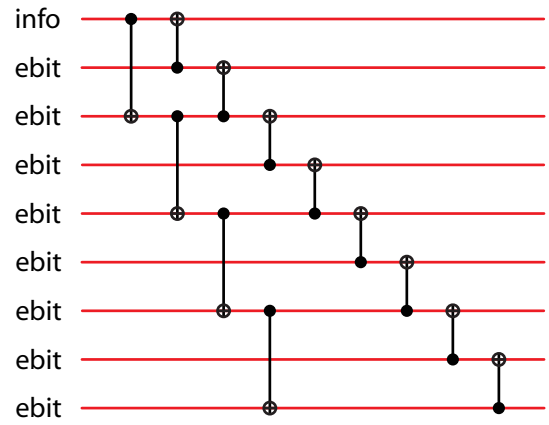

(a)

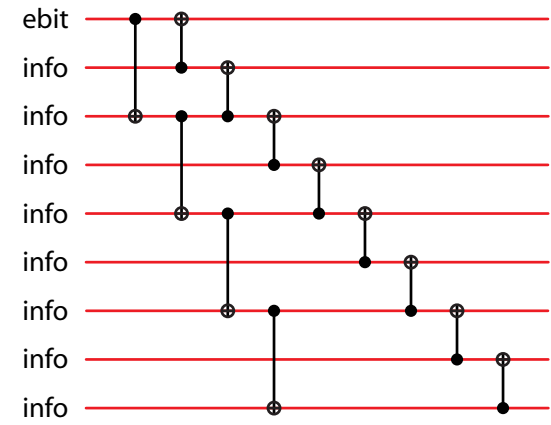

(b)

Fig. 1 (a) The encoder for a [[9,1,9;8]] EA repetition code consists of a periodic cascade of CNOT gates. The encoder for arbitrary $[[n, 1, n ; n-1]]$ EA repetition codes extends naturally from this design. We can implement these encoders with a simple quantum shift-register circuit that uses only one memory qubit 42. (b) Considering the circuit in (a) but changing the information qubit to an ebit and all of the ebits to information qubits gives the encoder for the dual of the EA repetition code, namely, the $[[9,8,2 ; 1]]$ EA accumulator code. This circuit naturally extends to encode the $[[n, n-1,2 ; 1]]$ EA accumulator codes. Simple variations of the above circuit encode the even $n$ repetition and accumulator EAQEC codes, and we discuss them in Section 4.2

Note that the commutation relations are as follows:

$$
\begin{aligned}
& g_{i} * g_{j}=0 \text { for } i \neq j, \\
& h_{i} * h_{j}=0 \text { for } i \neq j, \\
& g_{i} * h_{j}=0 \text { for } i \neq j, \\
& g_{i} * h_{i}=1 \text { for all } i .
\end{aligned}
$$

We say that $g_{i}$ and $h_{i}$ are symplectic partners for $i=1, \cdots, k+c$. The logical subgroup $\mathcal{L}$ of $\overline{\mathcal{G}}_{n}$ of the encoded state is

$$
\mathcal{L}=\left\langle g_{1}, \cdots, g_{k}, h_{1}, \cdots, h_{k}\right\rangle .
$$

The symplectic subgroup $\mathcal{S}_{S}$ of $\mathcal{S}^{\prime}$ is the subgroup generated by the $c$ pairs of symplectic partners of $\mathcal{S}^{\prime}$ :

$$
\mathcal{S}_{S}=\left\langle g_{k+1}, \cdots, g_{k+c}, h_{k+1}, \cdots, h_{k+c}\right\rangle
$$

The isotropic subgroup $\mathcal{S}_{I}$ of $\mathcal{S}^{\prime}$ is the subgroup generated by the generators $g_{i}$ of $\mathcal{S}^{\prime}$ such that $g_{i} * g=0$ for all $g$ in $\mathcal{S}^{\prime}$ :

$$
\mathcal{S}_{I}=\left\langle g_{k+c+1}, \cdots, g_{n}\right\rangle .
$$

Notice that $\mathcal{S}^{\prime}=\mathcal{S}_{S} \times \mathcal{S}_{I}$ in $\overline{\mathcal{G}}_{n}$. The minimum distance $d$ of the EAQEC code is the minimum weight of any element in $\left(\phi\left(\mathcal{S}^{\prime}\right)\right)^{\perp} \backslash \phi\left(\mathcal{S}_{I}\right)$.

\section{Duality in Entanglement-Assisted Quantum Codes}

\subsection{The Dual of an Entanglement Assisted Quantum Code}

The dual of an $[[n, k, d ; c]]$ EAQEC code, defined by a simplified stabilizer group $\mathcal{S}^{\prime}=\mathcal{S}_{S} \times \mathcal{S}_{I}$ and a logical group $\mathcal{L}$, is the $\left[\left[n, c, d^{\prime} ; k\right]\right]$ EAQEC code with $\mathcal{L} \times \mathcal{S}_{I}$ being the simplified stabilizer group and $\mathcal{S}_{S}$ being the logical group for some minimum distance $d^{\prime}[22$.

When $c=0$, the code is a standard stabilizer code. This case is not a concern of this paper.

When $c=n-k$, we call such a code a maximal-entanglement EAQEC code. In this case, $\mathcal{S}_{I}$ is the trivial group that contains only the identity, and the simplified stabilizer group is $\mathcal{S}_{S}$. Its dual code is a maximal-entanglement EAQEC code defined by the logical group $\mathcal{L}$. As an example, consider the class of $[[n, 1, n ; n-1]]$ EA repetition codes for $n$ odd [20. The two logical operators for the one logical qubit of this code are as follows:

$$
\begin{array}{llllll}
X & X & X & \cdots & X & X \\
Z & Z & Z & \cdots & Z & Z
\end{array}
$$


The simplified stabilizer generators are as follows:

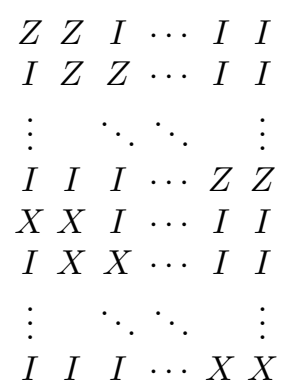

One can determine the symplectic pairs by performing a symplectic Gram-Schmidt orthogonalization of the above operators 41. If we interchange the roles of the stabilizer subgroup and the logical operator subgroup, we obtain an $[[n, n-1,2 ; 1]]$ EA accumulator code.

To make this more precise, consider the encoding circuits in Figure 1. The circuit in Figure 1(a) is the encoder of a $[[9,1,9 ; 8]]$ EA repetition code. Swapping the information qubit for an ebit and all of the ebits for information qubits gives the encoder of Figure 1(b), which encodes a $[[9,8,2 ; 1]]$ EA accumulator code. To illustrate that the circuit is working as expected, let us consider it acting on the first five qubits only (just to simplify the analysis). Inputting the following two operators at the information qubit slot

\section{$X I I I I$ \\ Z IIIII}

gives the following two logical operators:

$X X X X X$

$Z Z Z Z Z$

and these operators match the form of the logical operators for the repetition code in (5). Inputting the following operators at the ebit slots
$\begin{array}{lllll}I & X & I & I & I\end{array}$
$\begin{array}{lllll}I & Z & I & I & I\end{array}$
$\begin{array}{lllll}I & I & X & I & I\end{array}$
$\begin{array}{lllll}I & I & Z & I & I\end{array}$
$\begin{array}{lllll}I & I & I & X & I\end{array}$,
$\begin{array}{lllll}I & I & I & Z & I\end{array}$
$\begin{array}{lllll}I & I & I & I & X\end{array}$
$\begin{array}{lllll}I & I & I & I & Z\end{array}$

gives the following operators

$$
\begin{array}{ccccc}
X & X & I & I & I \\
I & Z & Z & Z & Z \\
I & X & X & X & X \\
Z & Z & I & I & I \\
I & I & X & X & I \\
I & I & I & Z & Z \\
I & I & I & X & X \\
I & I & Z & Z & I
\end{array}
$$

which we can transform by row operations to be the same as the operators in (6).

\subsection{The MacWilliams Identity}

The MacWilliams identity for general quantum codes can be obtained by applying the Poisson summation formula from the theory of orthogonal groups [18,22]. We restate Theorem 2 and Corollary 3 in [22] here. 


\section{Theorem 1 (Theorem 2 of [22]) Suppose}

$$
W_{V}(x, y)=\sum_{w=0}^{n} B_{w} x^{n-w} y^{w}
$$

and

$$
W_{V^{\perp}}(x, y)=\sum_{w^{\prime}=0}^{n} A_{w^{\prime}} x^{n-w^{\prime}} y^{w^{\prime}}
$$

are the weight enumerators of subgroup $V$ of $\overline{\mathcal{G}}_{n}$ and its orthogonal group $V^{\perp}$ in $\overline{\mathcal{G}}_{n}$, respectively. Then

$$
W_{V}(x, y)=\frac{1}{\left|V^{\perp}\right|} W_{V^{\perp}}(x+3 y, x-y)
$$

or

$$
B_{w}=\frac{1}{\left|V^{\perp}\right|} \sum_{w^{\prime}=0}^{n} P_{w}\left(w^{\prime}, n\right) A_{w^{\prime}}, \text { for } w=0, \cdots, n
$$

where $P_{w}\left(w^{\prime}, n\right)=\sum_{u=0}^{w}(-1)^{u} 3^{w-u}\left(\begin{array}{c}w^{\prime} \\ u\end{array}\right)\left(\begin{array}{c}n-w^{\prime} \\ w-u\end{array}\right)$ is the Krawtchouk polynomial 24.

Corollary 1 (Corollary 3 of [22]) The MacWilliams identities for EAQEC codes are as follows:

$$
\begin{aligned}
W_{\mathcal{L} \times \mathcal{S}_{I}}(x, y) & =\frac{1}{\left|\mathcal{S}_{S} \times \mathcal{S}_{I}\right|} W_{\mathcal{S}_{S} \times \mathcal{S}_{I}}(x+3 y, x-y) \\
W_{\mathcal{S}_{I}}(x, y) & =\frac{1}{\left|\mathcal{L} \times \mathcal{S}_{S} \times \mathcal{S}_{I}\right|} W_{\mathcal{L} \times \mathcal{S}_{S} \times \mathcal{S}_{I}}(x+3 y, x-y) .
\end{aligned}
$$

In the case of maximal-entanglement EAQEC codes, $\mathcal{S}_{I}$ is the trivial group and there is no degeneracy. If we exchange the roles of $\mathcal{S}_{S}$ and $\mathcal{L}$, we obtain an $\left[\left[n, n-k, d^{\prime} ; k\right]\right]$ EAQEC code, which is the dual of the original $[[n, k, d ; n-k]]$ EAQEC code. The minimum distance $d^{\prime}$ of this $\left[\left[n, n-k, d^{\prime} ; k\right]\right]$ EAQEC code is the minimum weight of a nontrivial element in $\mathcal{L}^{\perp}=\mathcal{S}_{S}$. Thus $d^{\prime}$ can be determined from the MacWilliams identity and the weight enumerator of the $[[n, k, d ; n-k]]$ EAQEC code, as in the following example.

Example 1 The dual of the $[[n, 1, n ; n-1]]$ repetition code is the $[[n, n-1,2 ; 1]]$ accumulator code whenever $n$ is odd. The coefficients of $W_{\mathcal{L}}(x, y)=\sum_{w=0}^{n} B_{w} x^{n-w} y^{w}$ for the odd- $n[[n, 1, n ; n-1]]$ repetition code are $B_{(n)} \triangleq\left(B_{0}, \cdots, B_{n}\right)=(1,0,0, \cdots, 0,3)$. Let $A_{(n)}=\left(A_{0}, \cdots, A_{n}\right)$. Using the MacWilliams identity, we obtain the weight enumerators $W_{\mathcal{S}_{S}}(x, y)=\sum_{w=0}^{n} A_{w} x^{n-w} y^{w}$ of these dual EAQEC codes:

$$
\begin{aligned}
A_{(3)}= & (1,0,9,6), \\
B_{(3)}= & (1,0,0,3), \\
A_{(5)}= & (1,0,30,60,105,60), \\
B_{(5)}= & (1,0,0,0,0,3), \\
A_{(7)}= & (1,0,63,210,735,1260,1281,546), \\
B_{(7)}= & (1,0,0,0,0,0,0,3), \\
A_{(9)}= & (1,0,108,504,2646,7560,15372, \\
& 19656,14769,4920), \\
B_{(9)}= & (1,0,0,0,0,0,0,0,0,3), \\
A_{(11)}= & (1,0,165,990,6930,27720,84546, \\
& 180180,270765,270600,162393,44286), \\
B_{(11)}= & (1,0,0,0,0,0,0,0,0,0,0,3) .
\end{aligned}
$$




\subsection{Linear Programming Bounds for EAQEC Codes}

The significance of the MacWilliams identities is that linear programming techniques can be applied to find upper bounds on the minimum distance of EAQEC codes 22. We have the MacWilliams identities (9) and (10) in Corollary 1. Suppose the weight enumerators of $\mathcal{S}_{S} \times \mathcal{S}_{I}, \mathcal{L} \times \mathcal{S}_{I}, \mathcal{S}_{I}$, and $\mathcal{L} \times \mathcal{S}_{S} \times \mathcal{S}_{I}$ are $W_{\mathcal{S}_{S} \times \mathcal{S}_{I}}(x, y)=\sum_{w=0}^{n} A_{w} x^{n-w} y^{w}, W_{\mathcal{L} \times \mathcal{S}_{I}}(x, y)=\sum_{w=0}^{n} B_{w} x^{n-w} y^{w}, W_{\mathcal{S}_{I}}(x, y)=\sum_{w=0}^{n} C_{w} x^{n-w} y^{w}$, and $W_{\mathcal{L} \times \mathcal{S}_{S} \times \mathcal{S}_{I}}(x, y)=\sum_{w=0}^{n} D_{w} x^{n-w} y^{w}$, respectively.

Since the minimum distance of an EAQEC code is the minimum weight of any element in $\left(\mathcal{L} \times \mathcal{S}_{I}\right) \backslash \mathcal{S}_{I}$, it is the minimum nonzero integer $w$ such that $B_{w}-C_{w}>0$. With constraints on $B_{w}$ 's and $C_{w}$ 's, we can find the linear programming bound on the minimum distance of the EAQEC code. To sum up, we have the following constraints:

$$
\begin{aligned}
& A_{0}=B_{0}=C_{0}=D_{0}=1 ; \\
& A_{w} \geq 0, B_{w} \geq 0, C_{w} \geq 0, D_{w} \geq 0, \text { for } w=1, \ldots, n ; \\
& A_{w} \leq\left|\mathcal{S}_{S} \times \mathcal{S}_{I}\right|, B_{w} \leq\left|\mathcal{L} \times \mathcal{S}_{I}\right|, \text { for } w=1, \ldots, n ; \\
& C_{w} \leq\left|\mathcal{S}_{I}\right|, D_{w} \leq\left|\mathcal{L} \times \mathcal{S}_{\mathcal{S}} \times \mathcal{S}_{I}\right|, \text { for } w=1, \ldots, n ; \\
& D_{w} \geq A_{w}, D_{w} \geq B_{w}, D_{w} \geq C_{w}, \text { for } w=1, \ldots, n ; \\
& A_{w} \geq C_{w}, B_{w} \geq C_{w}, \text { for } w=1, \ldots, n ; \\
& \sum_{w=0}^{n} A_{w}=\left|\mathcal{S}_{S} \times \mathcal{S}_{I}\right|, \sum_{w=0}^{n} B_{w}=\left|\mathcal{L} \times \mathcal{S}_{I}\right|, \\
& \sum_{w=0}^{n} C_{w}=\left|\mathcal{S}_{I}\right|, \sum_{w=0}^{n} D_{w}=\left|\mathcal{L} \times \mathcal{S}_{\mathcal{S}} \times \mathcal{S}_{I}\right| ; \\
& B_{w}=\frac{1}{\left|\mathcal{S}_{S} \times \mathcal{S}_{I}\right|} \sum_{w^{\prime}=0}^{n} P_{w}\left(w^{\prime}, n\right) A_{w^{\prime}}, \text { for } w=0, \ldots, n ; \\
& D_{w}=\frac{1}{\left|\mathcal{S}_{I}\right|} \sum_{w^{\prime}=0}^{n} P_{w}\left(w^{\prime}, n\right) C_{w^{\prime}}, \text { for } w=0, \ldots, n ; \\
& B_{w}=C_{w}, \text { for } w=1, \ldots, d-1 ;
\end{aligned}
$$

If we cannot find any solutions to the integer programming problem with the above constraints for a certain $d$, this result implies that there is no $[[n, k, d ; c]]$ EAQEC code. If $d^{*}$ is the smallest such $d$, then $d^{*}-1$ is an upper bound on the minimum distance of an $[[n, k, d ; c]]$ EAQEC code. This is the linear programming bound for EAQEC codes with $0<c<n-k$. If we replace the constraints

$$
B_{w}=C_{w}, \text { for } w=1, \ldots, d-1,
$$

with

$$
A_{w}=C_{w}, \text { for } w=1, \ldots, d-1,
$$

this gives the constraints of the linear programming bound on the minimum distance of the $[[n, c, d ; k]]$ dual code.

Example 2 Consider the $[[8,3,5 ; 5]]$ EAQEC code from the random optimization algorithm in Ref. [20]. The linear programming bound shows that there is no $[[8,3, d ; 5]]$ EAQEC code with $d>5$, and thus the $[[8,3,5 ; 5]]$ code is optimal.

Example 3 Consider the [[15, 7, 6; 8]] EAQEC code from the random optimization algorithm in Ref. [20]. The linear programming bound shows that no $[[15,7, d ; 8]]$ EAQEC code with $d>7$ exists; however, it does not rule out the existence of a $[[15,7,7 ; 8]]$ code.

Example 4 The linear programming bound of the $[[7,1 ; 4]]$ code is $d \leq 6$, which is the same as the singleton bound. Hence the $[[7,1,5 ; 4]]$ EAQEC code from the encoding optimization algorithm in Example 4 of [20] does not achieve the linear programming bound. It follows that the $[[7,1,5 ; 5]]$ EAQEC code does not achieve the upper bound either. 
Example 5 The linear programming bound of the $[[8,3 ; 3]]$ EAQEC code is $d \leq 4$, which improves the singleton bound: $d \leq 5$. Hence the $[[8,3,4 ; 3]]$ EAQEC code from the encoding optimization algorithm in Example 8 of $[20$ is optimal. On the other hand, the linear programming bound of the $[[8,3 ; 4]]$ EAQEC code is $d \leq 5$, which is the same as the singleton bound. Hence the $[[8,3,4 ; 4]]$ EAQEC code does not achieve the upper bound.

Example 6 The linear programming bounds of $[[9,1 ; c]]$ EAQEC codes for $c=3,4,5$ are $d \leq 8$; however, the singleton bounds are $d \leq 6, d \leq 7$, and $d \leq 7$, respectively.

Remark 1 From these three examples, we can determine that the linear programming bound might be better or worse than the Singleton bound when $0<c<n-k$.

\section{Bounds on EAQEC Codes}

Table I of Ref. 22 establishes lower and upper bounds on the minimum distance of maximal-entanglement EAQEC codes with length $n \leq 15$. This section provides a detailed justification for these results. We begin by discussing the existence of arbitrary EAQEC codes, followed by some specific EAQEC code constructions.

The existence of an $[[n, k, d]]$ stabilizer code implies the existence of an $\left[\left[n, k, d^{\prime} \geq d ; c\right]\right]$ EAQEC code, since we can replace ancilla qubits with ebits and then optimize the encoding operator [20. Therefore, the lower bound on the minimum distance of regular stabilizer codes [7] can be applied here. Similarly, the existence of an $[[n, k, d ; c]]$ EAQEC code where $c<n-k$ implies the existence of an $\left[\left[n, k, d^{\prime} \geq d ; c^{\prime}>c\right]\right]$ EAQEC code. This establishes the existence of many $[[n, k ; c]]$ EAQEC codes.

\subsection{Gilbert-Varshamov Bound for EAQEC Codes}

Consider the stabilizer group $\mathcal{S}$ of an $[[n, k, d ; c]]$ EAQEC code, which is a subgroup of the Pauli group $\mathcal{G}^{n+c}$. We consider only error operators in the group $\mathcal{G}^{n}$ because the entanglement-assisted paradigm assumes that the ebits on Bob's side of the channel are not subject to errors. The EAQEC code is defined in an $(n+c)$-qubit space, but only the first $n$ qubits suffer from errors. Following the argument of the quantum Gilbert-Varshamov bound 13, we obtain the Gilbert-Varshamov bound for EAQEC codes. However, we will show that there are maximal-entanglement EAQEC codes with minimum distance higher than this bound for $n \leq 15$.

Theorem 2 Given $n, d, c$, let

$$
k=\left\lceil\log _{2}\left(\frac{2^{n+c}}{\sum_{j=0}^{d-1} 3^{j}\left(\begin{array}{c}
n \\
j
\end{array}\right)}\right)\right\rceil .
$$

The Gilbert-Varshamov bound for EAQEC codes states that if $0 \leq k \leq n-c$, then there exists an $[[n, k, d ; c]]$ EAQEC code. Equivalently,

$$
\sum_{j=0}^{d-1} 3^{j}\left(\begin{array}{l}
n \\
j
\end{array}\right) 2^{k} \geq 2^{n+c} .
$$

Most of the lower bounds in Table 1 of Ref. 22] are slightly higher than the Gilbert-Varshamov bounds for $n \leq 15$.

\subsection{Maximal-Entanglement EAQEC Repetition and Accumulator Codes for Even $n$}

Lai and Brun proposed a construction of [[n,1,n;n-1]] EA repetition codes for $n$ odd in Ref. 20]. In the case of even $n$, that construction gives a series of $[[n, 0, n ; n-2]]$ EA repetition codes with no information qubits.

In this section, we construct $[[n, 1, n-1 ; n-1]]$ EA repetition codes for $n$ even. We obtained these codes both with the techniques from Ref. 20] and by realizing that the encoding circuit in Figure 1 can encode 
them. The duals of these codes are the $[[n, n-1,1 ; 1]]$ EA accumulator codes for even $n$. Theorems 4 and 5 show that both of these code constructions are optimal, in the sense that $[[n, 1, n ; n-1]]$ and $[[n, n-1,2 ; 1]]$ EAQEC codes do not exist for even $n$. Thus, the results here complete our understanding of the dual classes of EA repetition and accumulator codes for arbitrary $n$.

Theorem 3 There are $[[n, 1, n-1 ; n-1]]$ EA repetition codes for $n$ even. The duals of these codes are the $[[n, n-1,1 ; 1]]$ EA accumulator codes.

Proof Suppose $H_{(n-1)}$ is an $(n-2) \times(n-1)$ parity-check matrix of a classical $[n-1,1, n-1]$ binary repetition code:

$$
H_{(n-1)}=\left[\begin{array}{cccccc}
1 & 1 & 0 & \cdots & 0 & 0 \\
0 & 1 & 1 & \cdots & 0 & 0 \\
\vdots & & \ddots & \ddots & & \vdots \\
0 & \cdots & \cdots & 0 & 1 & 1
\end{array}\right] \text {. }
$$

We define two $(n-1) \times n$ matrices

$$
H_{1}=\left[\begin{array}{lllll}
0 & & & & \\
\vdots & H_{(n-1)} & & \\
0 & & & & \\
1 & 1 & \cdots & 1 & 0
\end{array}\right]
$$

and

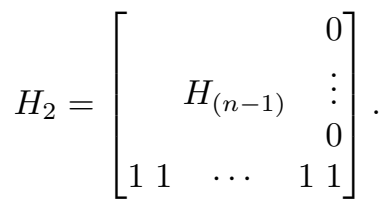

Consider a simplified check matrix of the form

$$
H^{\prime}=\left[\begin{array}{cc}
O & H_{1} \\
H_{2} & O
\end{array}\right]
$$

Consider the matrix $H_{1} H_{2}^{T}$. We have that

$$
\left[H_{1} H_{2}^{T}\right]_{i, j}=\left\{\begin{array}{l}
\text { if } i=j \text { for } j=1, \cdots, n-1, \text { or } \\
1, \quad i=j-2 \text { for } j=3, \cdots, n-2 \\
0, \text { else. }
\end{array}\right.
$$

For example, when $n=6$,

$$
H_{1} H_{2}^{T}=\left[\begin{array}{lllll}
1 & 0 & 1 & 0 & 0 \\
0 & 1 & 0 & 1 & 0 \\
0 & 0 & 1 & 0 & 0 \\
0 & 0 & 0 & 1 & 0 \\
0 & 0 & 0 & 0 & 1
\end{array}\right] .
$$

Thus the number of symplectic pairs in $H^{\prime}$ is as follows [44]:

$$
\frac{1}{2} \operatorname{rank}\left(H^{\prime} \Lambda H^{\prime T}\right)=\operatorname{rank}\left(H_{1} H_{2}^{T}\right)=n-1 .
$$

The simplified logical matrix is

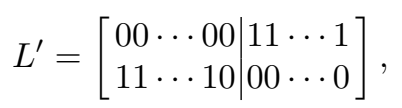

which implies the minimum distance is $n-1$. Therefore, $H^{\prime}$ and $L^{\prime}$ define an $[[n, 1, n-1 ; n-1]]$ EAQEC code. One obtains the dual $[[n, n-1,1 ; 1]]$ codes simply by swapping the roles of the logical matrix and the simplified check matrix.

This completes the family of EA repetition and accumulator codes for any $n$. The encoding circuit of Figure 1 encodes these even- $n$ repetition codes with the exception that the last qubit is removed, the last CNOT in the first string does not act, and the last CNOT in the second string does not act. 
Example 7 The coefficients of $W_{\mathcal{L}}(x, y)=\sum_{w=0}^{n} B_{w} x^{n-w} y^{w}$ for the even- $n[[n, 1, n-1 ; n-1]]$ EA repetition code are $B_{(n)}=(1,0, \cdots, 0,1,2)$. Using the MacWilliams identity, we obtain the weight enumerators $W_{\mathcal{S}_{S}}(x, y)=\sum_{w=0}^{n} A_{w} x^{n-w} y^{w}$ of these dual even- $n$ EAQEC codes:

$$
\begin{aligned}
A_{(4)}= & (1,1,15,27,20), \\
B_{(4)}= & (1,0,0,1,2), \\
A_{(6)}= & (1,1,40,130,305,365,182), \\
B_{(6)}= & (1,0,0,0,0,1,2), \\
A_{(8)}= & (1,1,77,357,1435,3395,5103,4375,1640), \\
B_{(8)}= & (1,0,0,0,0,0,0,1,2), \\
A_{(10)}= & (1,1,126,756,4326,15246,38304,65604, \\
& 73809,49209,14762), \\
B_{(10)}= & (1,0,0,0,0,0,0,0,0,1,2), \\
A_{(12)}= & (1,1,187,1375,10230,47850,168630,432894, \\
& 811965,1082565,974303,531443,132860), \\
B_{(12)}= & (1,0,0,0,0,0,0,0,0,0,0,1,2) .
\end{aligned}
$$

The even- $n[[n, 1, n-1 ; n-1]]$ EA repetition codes do not saturate the quantum singleton bound or the linear programming bounds. Were an even- $n[[n, 1, n ; n-1]]$ code to exist, it would have a weight enumerator $W_{\mathcal{L} \times \mathcal{S}_{I}}(x, y)=\sum_{w=0}^{n} B_{w} x^{n-w} y^{w}$ with $B_{0}=1, B_{n}=3$, and $B_{w}=0$ for $w \neq 0, n$. The weight enumerator of its dual would also have the coefficients

$$
A_{w}=\frac{1}{4}\left(3^{w}+3(-1)^{w}\right)\left(\begin{array}{l}
n \\
w
\end{array}\right)
$$

which are positive integers for $w=0, \cdots, n$. It would only be able to correct up to $\left\lfloor\frac{n-1}{2}\right\rfloor$ channel qubit errors, which is the same number of errors that our even- $n$ repetition codes can correct. We prove below that even- $n[[n, 1, n ; n-1]]$ EAQEC codes do not exist, and thus our even- $n$ repetition codes from Theorem 3 are optimal.

Theorem 4 There is no $[[n, 1, n ; n-1]]$ EAQEC code for $n$ even.

Proof We prove this theorem by contradiction. Suppose there is an $[[n, 1, n ; n-1]]$ EAQEC code for $n$ even with a $2 \times 2 n$ logical matrix

$$
\left[\begin{array}{c|c}
u^{1} & v^{1} \\
u^{2} & v^{2}
\end{array}\right]
$$

where $u^{1}, u^{2}, v^{1}$, and $v^{2}$ are binary row vectors of length $n$. These vectors should satisfy the following condition in order for the above matrix to be a valid logical matrix:

$$
u^{1} \cdot v^{2}+u^{2} \cdot v^{1}=1 \bmod 2 .
$$

Let $\operatorname{gw}(\cdot)$ be the "general weight" function defined by

$$
\operatorname{gw}(u \mid v) \equiv \sum_{i: u_{i}=1 \text { or } v_{i}=1} 1,
$$

where $u_{i}$ denotes the $i^{\text {th }}$ bit of the binary $n$-tuple $u$. The above binary vectors should satisfy the further constraints

$$
\operatorname{gw}\left(u^{1} \mid v^{1}\right)=\operatorname{gw}\left(u^{2} \mid v^{2}\right)=\operatorname{gw}\left(u^{1}+u^{2} \mid v^{1}+v^{2}\right)=n,
$$

in order for the code to have distance $n$ as claimed.

We now use the above constraints to obtain a contradiction. We first partition the first row of the matrix into subsets $A, B$, and $C$ of $X, Y$, and $Z$ operators, respectively. There should not be any identity operators in the first row in order for the code to have distance $n$. Up to permutations on the qubits (under which the distance is invariant), the logical matrix has the following form:

$$
\left[\begin{array}{ccc|ccc}
\mathbf{1} & \mathbf{1} & \mathbf{0} & \mathbf{0} & \mathbf{1} & \mathbf{1} \\
u_{A}^{2} & u_{B}^{2} & u_{C}^{2} & v_{A}^{2} & v_{B}^{2} & v_{C}^{2}
\end{array}\right]
$$


where $\mathbf{1}$ is a vector of all ones, $\mathbf{0}$ is a vector of all zeros, and we have split up the vector $\left(u^{2} \mid v^{2}\right)$ into different components corresponding to the subsets $A, B$, and $C$. Consider the vector $u_{A}^{2}$. Suppose that a component $\left(u_{A}^{2}\right)_{i}=0$. Then $\left(v_{A}^{2}\right)_{i}$ should equal 1 so that the code's distance is not less than $n$. Now suppose that $\left(u_{A}^{2}\right)_{i}=1$. Then $\left(v_{A}^{2}\right)_{i}$ should also equal 1 so that the code's distance is not less than $n$. Otherwise, we could add $(1 \mid 0)$ to $\left(\left(u_{A}^{2}\right)_{i} \mid\left(v_{A}^{2}\right)_{i}\right)$ and obtain $(0 \mid 0)$ as a component of another logical operator, and such a result would imply that the code's distance is less than $n$. These steps imply that the logical matrix should have the following form:

$$
\left[\begin{array}{ccc|ccc}
\mathbf{1} & \mathbf{1} & \mathbf{0} & \mathbf{0} & \mathbf{1} & \mathbf{1} \\
u_{A}^{2} & u_{B}^{2} & u_{C}^{2} & \mathbf{1} & v_{B}^{2} & v_{C}^{2}
\end{array}\right]
$$

Similar reasoning with $v_{C}^{2}$ and $u_{C}^{2}$ implies that $u_{C}^{2}$ should equal $\mathbf{1}$, and the logical matrix should then have the following form:

$$
\left[\begin{array}{ccc|ccc}
\mathbf{1} & \mathbf{1} & \mathbf{0} & \mathbf{0} & \mathbf{1} & \mathbf{1} \\
u_{A}^{2} & u_{B}^{2} & \mathbf{1} & \mathbf{1} & v_{B}^{2} & v_{C}^{2}
\end{array}\right]
$$

Finally, consider the vector $u_{B}^{2}$. Suppose that a component $\left(u_{B}^{2}\right)_{i}=1$. Then $\left(v_{B}^{2}\right)_{i}$ should equal 0 so that the code's distance is not less than $n$. Otherwise, we could add (1|1) to $\left(\left(u_{B}^{2}\right)_{i} \mid\left(v_{B}^{2}\right)_{i}\right)$ and obtain $(0 \mid 0)$ as a component of another logical operator, and such a result would imply that the code's distance is less than $n$. Now suppose that $\left(u_{B}^{2}\right)_{i}=0$. Then $\left(v_{B}^{2}\right)_{i}$ should equal 1 , by reasoning similar to the above. Thus, the logical matrix should have the following form in order for the code's distance to be equal to $n$ :

$$
\left[\begin{array}{rrr|rrr}
\mathbf{1} & \mathbf{1} & \mathbf{0} & \mathbf{0} & \mathbf{1} & \mathbf{1} \\
u_{A}^{2} & u_{B}^{2} & \mathbf{1} & \mathbf{1} & \bar{u}_{B}^{2} & v_{C}^{2}
\end{array}\right]
$$

where $\bar{u}_{B}^{2}$ is the binary complement of $u_{B}^{2}$. Now, the symplectic product of the above two vectors is

$$
(|A|+|B|+|C|) \bmod 2=n \bmod 2=0,
$$

which contradicts the assumption that the original matrix is a valid logical matrix.

Theorem 5 There is no $[[n, n-1,2 ; 1]]$ EAQEC code for $n$ even.

Proof We prove the theorem by contradiction, in a fashion similar to the previous theorem. Suppose there is an $[[n, n-1,2 ; 1]]$ EAQEC code for $n$ even, and suppose its $2 \times 2 n$ simplified check matrix $\left[H_{X} \mid H_{Z}\right]$ has the form

$$
\left[\begin{array}{l|l}
u^{1} & v^{1} \\
u^{2} & v^{2}
\end{array}\right]
$$

where $u^{1}, u^{2}, v^{1}$, and $v^{2}$ are binary vectors of length $n$. These vectors should satisfy the following condition in order for the above matrix to be a simplified check matrix of a maximal-entanglement EAQEC code with one ebit:

$$
u^{1} \cdot v^{2}+u^{2} \cdot v^{1}=1 \bmod 2 .
$$

We now partition the first row of the simplified check matrix into subsets $A, B$, and $C$ of $X, Y$, and $Z$ operators, respectively. Up to permutations on the qubits (under which the distance is invariant), the simplified check matrix has the following form:

$$
\left[\begin{array}{ccc|ccc}
\mathbf{1} & \mathbf{1} & \mathbf{0} & \mathbf{0} & \mathbf{1} & \mathbf{1} \\
u_{A}^{2} & u_{B}^{2} & u_{C}^{2} & v_{A}^{2} & v_{B}^{2} & v_{C}^{2}
\end{array}\right]
$$

where we have split up the vector $\left(u^{2} \mid v^{2}\right)$ into different components corresponding to the subsets $A$, $B$, and $C$. The code has minimum distance two by assumption, and is non-degenerate because it is a maximal-entanglement EAQEC code. Therefore, no column of the above matrix should be equal to the all-zeros vector. Were it not so, then the code would not be able to detect every single-qubit $X$ or $Z$ error and would not have distance two as claimed. These constraints restrict the simplified check matrix to have the following form:

$$
\left[\begin{array}{ccc|ccc}
\mathbf{1} & \mathbf{1} & \mathbf{0} & \mathbf{0} & \mathbf{1} & \mathbf{1} \\
u_{A}^{2} & u_{B}^{2} & \mathbf{1} & \mathbf{1} & v_{B}^{2} & v_{C}^{2}
\end{array}\right]
$$


Also, no column of the entrywise sum of the matrices to the left and right of the vertical bar should be equal to the all-zeros vector. Were it not so, then the code would not be able to detect every single-qubit $Y$ error and would not have distance two as claimed. These constraints further restrict the simplified check matrix to be as follows:

$$
\left[\begin{array}{rrr|rrr}
\mathbf{1} & \mathbf{1} & \mathbf{0} & \mathbf{0} & \mathbf{1} & \mathbf{1} \\
u_{A}^{2} & u_{B}^{2} & \mathbf{1} & \mathbf{1} & \bar{u}_{B}^{2} & v_{C}^{2}
\end{array}\right] .
$$

Now, the symplectic product of the above two vectors is

$$
(|A|+|B|+|C|) \bmod 2=n \bmod 2=0,
$$

which contradicts the assumption that the original matrix is a simplified check matrix for a maximalentanglement EAQEC code with one ebit.

The matching upper and lower bounds for $k=1$ in Table 1 of Ref. 22] are from the family of EA repetition codes.

Interestingly, observe that the non-existent logical matrix in 11 has the same form as the nonexistent simplified check matrix in (12). Were either type of code to exist, we would expect them to be duals of each other, but they both fail to exist because they cannot satisfy the dual constraints imposed on them.

\subsection{Existence of Other EAQEC Codes}

The following theorem is similar to Theorem 6 in Ref. 7]. It shows how to obtain new EAQEC codes from existing ones. These results are helpful in our search for lower bounds on the minimum distance of maximal-entanglement EAQEC codes.

Theorem 6 Suppose an $[[n, k, d ; c]]$ code exists. Then

1. An $[[n+1, k, d ; c+1]]$ code exists.

2. An $\left[\left[n, k-1, d^{\prime} \geq d ; c+1\right]\right]$ code exists.

Proof 1. Suppose $H=\left[H_{X} \mid H_{Z}\right]$ is a simplified check matrix of an $[[n, k, d ; c]]$ code. Then the simplified check matrix

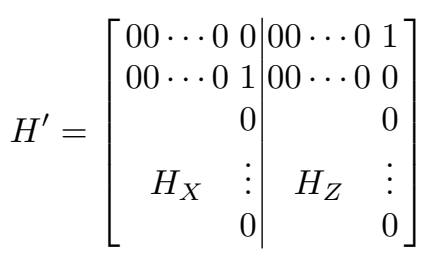

defines an $[[n+1, k, d ; c+1]]$ code. We have the stabilizer group $(\mathcal{S} \otimes I) \cup\left\{X_{n+1}, Z_{n+1}\right\}$, where $(\mathcal{S} \otimes I)=\{E \otimes I: E \in \mathcal{S}\}$.

2. It is obtained by moving a symplectic pair from the logical group to the stabilizer group.

\subsection{The Plotkin bound for EAQEC Codes}

The Plotkin bound for EAQEC codes is similar to the Plotkin bound for classical codes [24]. It is again helpful in our efforts to bound the minimum distance of maximal-entanglement EAQEC codes.

Theorem 7 The Plotkin bound for any $[[n, k, d ; c]]$ EAQEC code is

$$
d \leq \frac{3 n 2^{2 k-2}}{2^{2 k}-1}
$$


Proof The proof is based on the proof of the classical Plotkin bound in Ref. 24. Let $M=2^{n+k-c}$ be the number of operators in $\mathcal{L} \times \mathcal{S}_{I}$. We bound the quantity $\sum_{u, v \in \mathcal{L} \times \mathcal{S}_{I} \backslash \mathcal{S}_{I}} \operatorname{wt}(u \cdot v)$ in two different ways. First, we lower bound it. There are $M$ choices for $u$, and for each choice of $u$, there are $M-2^{n-k-c}$ choices for $v$ such that $u \cdot v \notin \mathcal{S}_{\mathcal{I}}$. Furthermore, for a code of minimum distance $d, \operatorname{wt}(u \cdot v) \geq d$ for any $u \cdot v \notin \mathcal{S}_{\mathcal{I}}$. So the following lower bound holds

$$
\begin{aligned}
M\left(M-2^{n-k-c}\right) d & \leq \sum_{u, v \in \mathcal{L} \times \mathcal{S}_{I}: u \cdot v \notin \mathcal{S}_{\mathcal{I}}} \mathrm{wt}(u \cdot v) \\
& \leq \sum_{u, v \in \mathcal{L} \times \mathcal{S}_{I}} \operatorname{wt}(u \cdot v) .
\end{aligned}
$$

The equality holds when $c=n-k$ because $S_{I}$ is trivial and $\operatorname{wt}(u \cdot v)=0$ if $u=v$. Now we obtain an upper bound on the quantity. We form an $M \times n$ matrix whose rows are the elements in the logical group $\mathcal{L} \times \mathcal{S}_{I}$. Let $m_{1}^{j}, m_{2}^{j}, m_{3}^{j}$, and $m_{4}^{j}$ be the number of $I, X, Y$, and $Z$ operators in column $j$ of this matrix, respectively. So the equality $\sum_{l=1}^{4} m_{l}^{j}=M$ holds for all $j \in\{1, \cdots, n\}$. Each choice of a particular Pauli operator and some other Pauli operator in the same column contributes exactly 2 to the $\operatorname{sum} \sum_{u, v \in \mathcal{L}} \operatorname{wt}(u \cdot v)$. Thus, the first equality below holds for this reason, and the second holds by applying $\sum_{l=1}^{4} m_{l}^{j}=M$ :

$$
\begin{aligned}
\sum_{u, v \in \mathcal{L} \times \mathcal{S}_{I}} \operatorname{wt}(u \cdot v) & =\sum_{j=1}^{n} \sum_{l=1}^{4} m_{l}^{j}\left(M-m_{l}^{j}\right) \\
& =\sum_{j=1}^{n}\left(M^{2}-\sum_{l=1}^{4}\left(m_{l}^{j}\right)^{2}\right) \\
& \leq \sum_{j=1}^{n}\left(M^{2}-\frac{M^{2}}{4}\right) \\
& =\frac{3 n}{4} M^{2} .
\end{aligned}
$$

The first inequality follows by applying $\sum_{l=1}^{4} m_{l}^{j} / 4=M / 4$ and convexity of the squaring function:

$$
(M / 4)^{2}=\left(\sum_{l=1}^{4} m_{l}^{j} / 4\right)^{2} \leq \sum_{l=1}^{4}\left(m_{l}^{j}\right)^{2} / 4
$$

Combining the lower and upper bounds gives us the EA Plotkin bound.

Since the proof is independent of the number of ebits $c$, the EA Plotkin bound applies to arbitrary EAQEC codes. However, note that $c$ does not appear in the bound, and consequently, this bound best describes the characteristics of maximal-entanglement EAQEC codes. However, for large $k$, the bound is approximately $\frac{3}{4} n$. Hence, this bound is useful only for small values of $k$.

Remark 2 The EA Plotkin bound has been improved in the case that an EAQEC code is "linear" [15]. For EAQEC codes corresponding to classical linear quaternary codes, the linear EA Plotkin bound is

$$
d \leq \frac{3 \cdot 2^{2 k}}{8\left(2^{2 k}-1\right)}(n+c+k)
$$

The Plotkin bound and the linear programming bound match for $k \leq 2$ and $n \leq 15$. For $k=3$ and $n=4,5,6,9,10,11,13,14,15$, they also match. For $k>3$, the Plotkin bound is not as tight as the linear programming bound, the Singleton bound, or the Hamming bound. 


\section{The Weight Enumerator Bound on the Block Error Probability under Maximum A Posteriori Decoding}

Since maximal-entanglement codes bear many similarities to classical codes, the block error probability when transmitting coded quantum information through the depolarizing channel can be upper bounded using the weight enumerator of a particular maximal-entanglement EAQEC code (similarly to the case for classical codes [30,25]). This "weight enumerator bound" gives an idea of the performance of maximumlikelihood decoding of an arbitrary maximal-entanglement EAQEC code. We can also determine the expected performance when decoding a random EAQEC code with a maximum likelihood decoding rule. Below, we determine these bounds and plot them for the maximal-entanglement repetition and accumulator EAQEC codes. The result is that these codes perform comparably to a random EA code with respect to this upper bound.

Theorem 8 Suppose that a sender transmits an $[[n, k ; n-k]]$ maximal-entanglement EAQEC code over a depolarizing channel with parameter $p$, and furthermore, that the receiver decodes this code according to a maximum a posteriori (MAP) decoding rule. Then we have the following upper bound on the block error probability $P_{B}$ :

$$
P_{B} \leq B(\gamma)-1
$$

where $B(z) \triangleq W_{\mathcal{L}}(1, z)$ is the weight enumerator of the maximal-entanglement EAQEC code and $\gamma$ is the "Bhattacharyya parameter" for the depolarizing channel:

$$
\gamma \equiv 2 \sqrt{\frac{p}{3}(1-p)}+\frac{2}{3} p
$$

Proof Let $U$ be a Clifford encoder for the $[[n, k ; n-k]]$ maximal-entanglement EAQEC code. The encoded state $|\bar{\psi}\rangle^{A B}$ is $|\bar{\psi}\rangle^{A B}=\left(U^{A} \otimes I^{B}\right)\left(|\phi\rangle \otimes\left(\left|\Phi_{+}\right\rangle^{A B}\right)^{\otimes(n-k)}\right)$. Then Alice transmits her qubits (entangled with Bob's qubits) through $n$ independent uses of a depolarizing channel $\mathcal{E}$ where

$$
\mathcal{E}(\rho)=(1-p) \rho+\frac{p}{3}(X \rho X+Y \rho Y+Z \rho Z)
$$

and $\rho$ is the density operator of a single qubit. We assume that $p<3 / 4$ because the channel is completely depolarizing when $p=3 / 4$. Suppose that an error operator $\tilde{E} \in \mathcal{G}^{n}$ occurs after the depolarizing channel, and that $\mathbf{s}^{\mathbf{x}}, \mathbf{s}^{\mathbf{z}}$ are the binary vector representations of the error syndrome. Both $\mathbf{s}^{\mathbf{x}}$ and $\mathbf{s}^{\mathbf{z}}$ are of length $(n-k)$, and Bob observes them by first decoding the qubits with a decoding unitary $U^{\dagger}$ and then performing Bell measurements on the ebits. This implies that

$$
\left(U^{\dagger} \tilde{E}\right)^{A} \otimes I^{B}|\bar{\psi}\rangle^{A B}=\tilde{L}_{0}|\phi\rangle \otimes\left(\left(X^{\mathbf{s}^{\mathbf{x}}} Z^{\mathbf{s}^{\mathbf{z}}}\right)^{A} \otimes I^{B}\right)\left(\left|\Phi_{+}\right\rangle^{A B}\right)^{\otimes n-k}
$$

and $\tilde{E}=U\left(\tilde{L}_{0} \otimes X^{\mathbf{s}^{\mathbf{x}}} Z^{\mathbf{s}^{\mathbf{z}}}\right) U^{\dagger}$ for some logical error $\tilde{L}_{0} \in \mathcal{L}_{0}$, where $\mathcal{L}_{0}$ is the set of unencoded logical operators. Poulin et al. devised a maximum a posteriori decoder for standard stabilizer codes [27], ${ }^{2}$ and we can modify their decoder to be a maximum a posteriori decoder $L_{\mathrm{MAP}}\left(\mathbf{s}^{\mathbf{x}}, \mathbf{s}^{\mathbf{z}}\right)$ for maximalentanglement EAQEC codes, where

$$
L_{\mathrm{MAP}}\left(\mathbf{s}^{\mathbf{x}}, \mathbf{s}^{\mathbf{z}}\right) \equiv \underset{L \in \mathcal{L}_{0}}{\arg \max } \operatorname{Pr}\left\{L \mid \mathbf{s}^{\mathbf{x}}, \mathbf{s}^{\mathbf{z}}\right\}
$$

This decoder selects the most likely error operator acting on the logical qubits, given the syndrome information $\mathbf{s}^{\mathbf{x}}$ and $\mathbf{s}^{\mathbf{z}}$. We can calculate the above conditional distribution by applying the Bayes rule to the joint distribution $\operatorname{Pr}\left\{L, \mathbf{s}^{\mathbf{x}}, \mathbf{s}^{\mathbf{z}}\right\}$ :

$$
\operatorname{Pr}\left\{L \mid \mathbf{s}^{\mathbf{x}}, \mathbf{s}^{\mathbf{z}}\right\}=\frac{\operatorname{Pr}\left\{L, \mathbf{s}^{\mathbf{x}}, \mathbf{s}^{\mathbf{z}}\right\}}{\sum_{L^{\prime}} \operatorname{Pr}\left\{L^{\prime}, \mathbf{s}^{\mathbf{x}}, \mathbf{s}^{\mathbf{z}}\right\}},
$$

\footnotetext{
2 Poulin et al. described their decoder as a "maximum-likelihood" decoder [27], but a careful study of it reveals that their decoder should more properly be called a maximum a posteriori decoder.
} 
where

$$
\begin{aligned}
\operatorname{Pr}\left\{L, \mathbf{s}^{\mathbf{x}}, \mathbf{s}^{\mathbf{z}}\right\}=\left.\operatorname{Pr}\{E\}\right|_{E=U\left(L \otimes X^{\mathbf{s}^{\mathbf{x}}} Z^{\mathbf{s}^{\mathbf{z}}}\right) U^{\dagger}} \\
=\left.\left.(1-p)^{n-\operatorname{wt}(E)}\left(\frac{p}{3}\right)^{\mathrm{wt}(E)}\right|_{E=U\left(L \otimes X^{\mathbf{s}^{\mathbf{x}}} Z^{\mathbf{s}^{\mathbf{z}}}\right) U^{\dagger}}\right|_{E=U\left(L \otimes X^{\mathbf{s}^{\mathbf{x}}} Z^{\mathbf{s}^{\mathbf{z}}}\right) U^{\dagger}} .
\end{aligned}
$$

The distribution $\sum_{L^{\prime}} \operatorname{Pr}\left\{L^{\prime}, \mathbf{s}^{\mathbf{x}}, \mathbf{s}^{\mathbf{z}}\right\}$ is fixed over all choices of $L$. Since $p<3 / 4 \Longleftrightarrow p /(3(1-p))<1$, the best choice of $L$ for the maximum a posteriori decoder $L_{\mathrm{MAP}}\left(\mathbf{s}^{\mathbf{x}}, \mathbf{s}^{\mathbf{z}}\right)$ is the one that selects a recovery operator $L^{-1}=L$ such that $E=U\left(L \otimes X^{\mathbf{s}^{x}} Z^{\mathbf{s}^{\mathbf{z}}}\right) U^{\dagger}$ has the minimum weight. This minimum weight decoder is similar to a classical minimum distance decoder.

Let $\mathcal{L}$ be the set of encoded logical operators and $\mathcal{S}^{\prime}$ be the set of simplified stabilizer generators. Given $E_{0} \in \mathcal{L}_{0}$, let

$$
Q\left(E_{0}\right) \equiv\left\{\mathbf{s}^{\mathbf{x}}, \mathbf{s}^{\mathbf{z}}: \operatorname{Pr}\left\{\tilde{L}_{0} E_{0}, \mathbf{s}^{\mathbf{x}}, \mathbf{s}^{\mathbf{z}}\right\} \geq \operatorname{Pr}\left\{\tilde{L}_{0}, \mathbf{s}^{\mathbf{x}}, \mathbf{s}^{\mathbf{z}}\right\}\right\} .
$$

We can now bound the probability $P_{B}\left(\tilde{L}_{0}\right)$ of a block error given that the error operator $\tilde{L}_{0}$ occurs under this decoding scheme:

$$
\begin{aligned}
P_{B}\left(\tilde{L}_{0}\right) & =\operatorname{Pr}\left\{\text { MAP decoder fails } \mid \tilde{L}_{0} \text { occurs }\right\} \\
& =\operatorname{Pr}\left\{L_{\mathrm{MAP}}\left(\mathbf{s}^{\mathbf{x}}, \mathbf{s}^{\mathbf{z}}\right) \neq \tilde{L}_{0}\right\} \\
& =\operatorname{Pr}\left\{\tilde{L}_{0} \cdot L_{\mathrm{MAP}}\left(\mathbf{s}^{\mathbf{x}}, \mathbf{s}^{\mathbf{z}}\right) \neq I\right\} \\
& =\operatorname{Pr}\left\{\tilde{L}_{0} \cdot L_{\mathrm{MAP}}\left(\mathbf{s}^{\mathbf{x}}, \mathbf{s}^{\mathbf{z}}\right) \in \mathcal{L}_{0} \backslash I\right\} \\
& =\sum_{E_{0} \in \mathcal{L}_{0} \backslash I} \operatorname{Pr}\left\{\tilde{L}_{0} \cdot L_{\mathrm{MAP}}\left(\mathbf{s}^{\mathbf{x}}, \mathbf{s}^{\mathbf{z}}\right)=E_{0}\right\} \\
& \leq \sum_{E_{0} \in \mathcal{L}_{0} \backslash I \mathbf{s}^{\mathbf{x}}, \mathbf{s}^{\mathbf{z}} \in Q\left(E_{0}\right)} \operatorname{Pr}\left\{\tilde{L}_{0}, \mathbf{s}^{\mathbf{x}}, \mathbf{s}^{\mathbf{z}}\right\} .
\end{aligned}
$$

Since $\sqrt{\frac{\operatorname{Pr}\left\{\tilde{L}_{0} E_{0}, \mathbf{s}^{\mathbf{x}}, \mathbf{s}^{\mathbf{z}}\right\}}{\operatorname{Pr}\left\{\tilde{L}_{0}, \mathbf{s}^{\mathbf{x}}, \mathbf{s}^{\mathbf{z}}\right\}}} \geq 1$ for $\mathbf{s}^{\mathbf{x}}, \mathbf{s}^{\mathbf{z}} \in Q\left(E_{0}\right)$, we can multiply each term in sum by this factor and then

$$
\begin{aligned}
P_{B}\left(\tilde{L}_{0}\right) & \leq \sum_{E_{0} \in \mathcal{L}_{0} \backslash I \mathbf{s}^{\mathbf{x}}, \mathbf{s}^{\mathbf{z}} \in Q\left(E_{0}\right)} \sqrt{\operatorname{Pr}\left\{\tilde{L}_{0}, \mathbf{s}^{\mathbf{x}}, \mathbf{s}^{\mathbf{z}}\right\} \operatorname{Pr}\left\{\tilde{L}_{0} E_{0}, \mathbf{s}^{\mathbf{x}}, \mathbf{s}^{\mathbf{z}}\right\}} \\
& \leq \sum_{E_{0} \in \mathcal{L}_{0} \backslash I} \sum_{\mathbf{s}^{\mathbf{x}}, \mathbf{s}^{\mathbf{z}} \in \mathbb{Z}_{2}^{n-k}} \sqrt{\operatorname{Pr}\left\{\tilde{L}_{0}, \mathbf{s}^{\mathbf{x}}, \mathbf{s}^{\mathbf{z}}\right\} \operatorname{Pr}\left\{\tilde{L}_{0} E_{0}, \mathbf{s}^{\mathbf{x}}, \mathbf{s}^{\mathbf{z}}\right\}} \\
& =\sum_{E \in \mathcal{L} \backslash I} \sum_{M \in \mathcal{S}^{\prime}} \sqrt{\operatorname{Pr}\{\tilde{L} M\} \operatorname{Pr}\{\tilde{L} E M\}} \\
& \leq \sum_{E \in \mathcal{L} \backslash I} \sum_{M \in \mathcal{G}^{n}} \sqrt{\operatorname{Pr}\{\tilde{L} M\} \operatorname{Pr}\{\tilde{L} E M\}}
\end{aligned}
$$

where $\tilde{L}=U\left(\tilde{L}_{0} \otimes I\right) U^{\dagger}, E=U E_{0} U^{\dagger}$, and $M=U\left(I \otimes X^{\mathbf{s}^{\mathbf{x}}} Z^{\mathbf{s}^{\mathbf{z}}}\right) U^{\dagger} \in \mathcal{S}^{\prime}$. Observe that

$$
\begin{aligned}
\sum_{M \in \mathcal{G}^{n}} \sqrt{\operatorname{Pr}\{\tilde{L} M\} \operatorname{Pr}\{\tilde{L} E M\}} & =\sum_{M \in \mathcal{G}^{n}} \sqrt{\operatorname{Pr}\{M\} \operatorname{Pr}\{\tilde{L} E \tilde{L} M\}} \\
& =\sum_{M \in \mathcal{G}^{n}} \prod_{i=1}^{n} \sqrt{\operatorname{Pr}\left\{(M)_{i}\right\} \operatorname{Pr}\left\{(\tilde{L})_{i}(E)_{i}(\tilde{L})_{i}(M)_{i}\right\}} \\
& =\prod_{i=1}^{n} \sum_{(M)_{i} \in \mathcal{G}} \sqrt{\operatorname{Pr}\left\{(M)_{i}\right\} \operatorname{Pr}\left\{(\tilde{L})_{i}(E)_{i}(\tilde{L})_{i}(M)_{i}\right\}}
\end{aligned}
$$


It holds that $(\tilde{L})_{i}(E)_{i}(\tilde{L})_{i} \neq I$ if $(E)_{i} \neq I$ and so

$$
\sum_{(M)_{i} \in \mathcal{G}} \sqrt{\operatorname{Pr}\left\{(M)_{i}\right\} \operatorname{Pr}\left\{(\tilde{L})_{i}(E)_{i}(\tilde{L})_{i}(M)_{i}\right\}}=2 \sqrt{\frac{p}{3}(1-p)}+\frac{2}{3} p=\gamma .
$$

Otherwise,

$$
\sum_{(M)_{i} \in \mathcal{G}} \sqrt{\operatorname{Pr}\left\{(M)_{i}\right\} \operatorname{Pr}\left\{(\tilde{L})_{i}(E)_{i}(\tilde{L})_{i}(M)_{i}\right\}}=1 .
$$

Consequently,

$$
\begin{aligned}
P_{B}\left(\tilde{L}_{0}\right) & \leq \sum_{E \in \mathcal{L} \backslash I} \gamma^{\mathrm{wt}(\tilde{L} E \tilde{L})} \\
& =\sum_{E \in \mathcal{L} \backslash I} \gamma^{\mathrm{wt}(E)} \\
& =B(\gamma)-1
\end{aligned}
$$

Therefore, the probability $P_{B}$ of a block error is bounded by $B(\gamma)-1$ when taking the expectation over all $\tilde{L}_{0}$.

The above theorem is similar to Theorem 7.5 in Ref. [25, which determines an upper bound on the block error probability when transmitting a classical linear code over a binary symmetric channel.

Theorem 9 Suppose that the sender transmits a random $[[n, k ; n-k]]$ maximal-entanglement EAQEC code over a depolarizing channel with parameter $p$ and furthermore that the receiver decodes this code according to a maximum a posteriori decoding rule. Let $U$ be the Clifford encoder for this code. Then we have the following upper bound on the expected block error probability $\bar{P}_{B}$ :

$$
\bar{P}_{B}=\mathbb{E}_{U}\left\{P_{B}\right\} \leq \frac{2^{2 k}-1}{2^{2 n}-1}\left((1+3 \gamma)^{n}-1\right),
$$

where $\gamma$ is the Bhattacharyya parameter defined in the previous theorem and the expectation is with respect to the choice of random code. In particular, if the rate $k / n$ satisfies the following upper bound:

$$
\frac{k}{n}<1-\frac{1}{2} \log _{2}(1+3 \gamma),
$$

then the error probability decreases exponentially to zero in the asymptotic limit.

Proof We first establish a method for choosing a random maximal-entanglement EAQEC code. A natural method for doing so is first to fix a basis of Pauli operators $X_{1}, Z_{1}, X_{2}, Z_{2}, \ldots, X_{n}, Z_{n}$, where the first $n-k$ anticommuting pairs correspond to the stabilizer operators for the $n-k$ ebits and the next $k$ anticommuting pairs correspond to the logical operators for the $k$ information qubits. We then select a Clifford unitary uniformly at random from the Clifford group (see Section VI-A-2 of Ref. 12] for a relatively straightforward algorithm for doing so) and apply it to the above fixed basis. This procedure produces $2 n$ encoded operators $\bar{X}_{1}, \bar{Z}_{1}, \bar{X}_{2}, \bar{Z}_{2}, \ldots, \bar{X}_{n}, \bar{Z}_{n}$ that specify a random maximalentanglement EAQEC code.

We now need to determine the expected weight enumerator $\mathbb{E}_{U}\{B(z)\}=\sum_{w=0}^{n} \mathbb{E}_{U}\left\{B_{w}\right\} z^{w}$ for such a random maximal-entanglement code. This will allow us to apply Theorem 8 to get an upper bound on the expected block error probability. Each coefficient $\mathbb{E}_{U}\left\{B_{w}\right\}$ corresponds to the expected number of Pauli operators of weight $w$ that belong to the logical operator group of a random EA code. Equivalently, it corresponds to the expected number of Pauli operators of weight $w$ that commute with the entanglement subgroup of a random code. First, let us consider $\mathbb{E}_{U}\left\{B_{0}\right\}$. The identity operator is the only Pauli operator with weight zero. It commutes with all operators with unit probability. Thus, $\mathbb{E}_{U}\left\{B_{0}\right\}=1$. Now, let us consider $\mathbb{E}_{U}\left\{B_{w}\right\}$ with $w \geq 1$. We first determine the probability that a Pauli operator $g$ with non-zero weight commutes with the $2(n-k)$ encoded operators $\bar{X}_{1}, \bar{Z}_{1}, \bar{X}_{2}, \bar{Z}_{2}$, $\ldots, \bar{X}_{n-k}, \bar{Z}_{n-k}$ for a random EA code. To simplify the calculation, observe that applying a uniformly random Clifford unitary to the operators $X_{1}, Z_{1}, X_{2}, Z_{2}, \ldots, X_{n-k}, Z_{n-k}$ and then determining the 
probability that a fixed operator $g$ commutes with all of them is actually the same as keeping the basis fixed and applying a random Clifford to the operator $g$ itself. This holds because

$$
C f C^{\dagger} g \pm g C f C^{\dagger}=0 \quad \Longleftrightarrow \quad f C^{\dagger} g C \pm C^{\dagger} g C f=0 .
$$

Then a uniform distribution on the Clifford unitaries takes this operator $g$ to an arbitrary Pauli operator $g^{\prime}$, and the distribution induced is just the uniform distribution on all of the $2^{2 n}-1 n$-qubit Pauli operators not equal to the identity (this reasoning is the same as that in Section VI-A-1 of Ref. [12]). At this point, the argument becomes purely combinatorial, and the only operators that commute with the above fixed basis are the ones with identity acting on the first $n-k$ qubits. Thus, there are $2^{2 k}-1$ Pauli operators besides the identity that commute with the fixed basis, and we conclude that the probability that a fixed Pauli operator $g$ with non-zero weight commutes with the random set $\bar{X}_{1}, \bar{Z}_{1}, \bar{X}_{2}, \bar{Z}_{2}, \ldots$, $\bar{X}_{n-k}, \bar{Z}_{n-k}$ is

$$
\frac{2^{2 k}-1}{2^{2 n}-1}
$$

Now we can calculate the expected number of operators that are in the logical subgroup. The number of Pauli operators with weight $w$ is $\left(\begin{array}{c}n \\ w\end{array}\right) 3^{w}$. Consequently, we have

$$
\mathbb{E}_{U}\left\{B_{w}\right\}=\frac{2^{2 k}-1}{2^{2 n}-1}\left(\begin{array}{c}
n \\
w
\end{array}\right) 3^{w}
$$

which implies

$$
\begin{aligned}
\mathbb{E}_{U}\{B(z)\}-\mathbb{E}_{U}\left\{B_{0}\right\} & =\sum_{w=1}^{n} \mathbb{E}_{U}\left\{B_{w}\right\} z^{w} \\
& =\frac{2^{2 k}-1}{2^{2 n}-1} \sum_{w=1}^{n}\left(\begin{array}{c}
n \\
w
\end{array}\right) 3^{w} z^{w} \\
& =\frac{2^{2 k}-1}{2^{2 n}-1}\left((1+3 z)^{n}-1\right) .
\end{aligned}
$$

Therefore, by exploiting the result in Theorem 8, an upper bound on the expected block error probability for general EAQEC codes with maximal entanglement is

$$
\begin{aligned}
\mathbb{E}_{U}\left\{P_{B}\right\} & \leq \bar{B}(\gamma)-\bar{B}_{0}, \\
& =\frac{2^{2 k}-1}{2^{2 n}-1}\left((1+3 \gamma)^{n}-1\right)
\end{aligned}
$$

We can drive the expected error probability to be arbitrarily low in the large $n$ and $k$ limit by ensuring that

$$
\frac{k}{n}<1-\frac{1}{2} \log _{2}(1+3 \gamma)
$$

This bound is not as tight as the EA hashing bound (the optimal limit), and Figure 2 displays how these two bounds differ.

We can plot the error probability bound in (13) as a function of $p$ for specific codes such as the repetition codes or the accumulator codes and then compare the results with the average error probability bound for a random code. Figure 3 provides such plots and compares their performance with a random EA code, with respect to these bounds. 


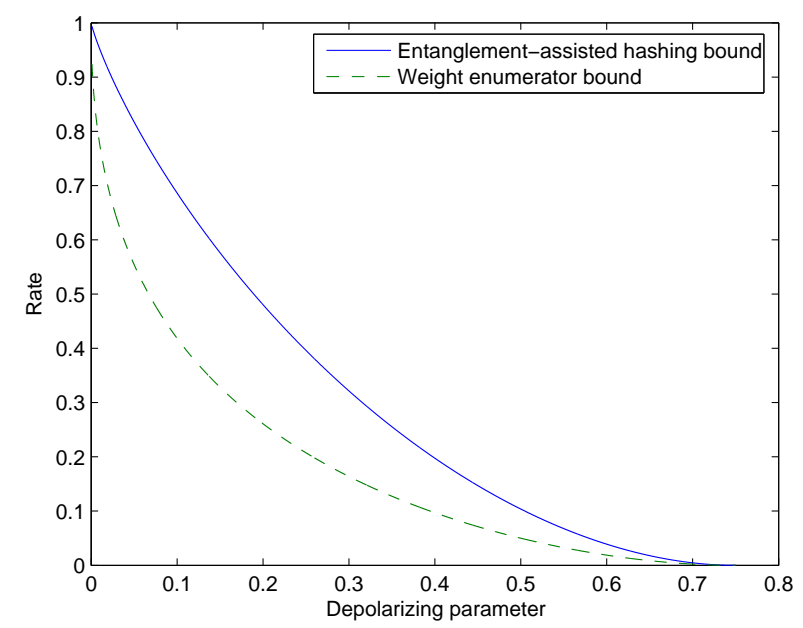

Fig. 2 The figure plots both the EA hashing bound $1-1 / 2\left[H_{2}(p)+p \log _{2} 3\right]$ from Ref. [3] and the "asymptotic weight enumerator bound" from [15 as a function of the depolarizing parameter. The two bounds become close for high depolarizing noise. Interestingly, the thresholds of the maximal-entanglement EA turbo codes from Ref. 40] are just shy of the asymptotic weight enumerator bound (see Figures 6(b) and 7(b) of that paper).

\section{Hashing Bounds for Pauli Channels}

The hashing bound of a quantum channel is an achievable rate for reliable quantum communication [2], and as such, it constitutes a lower bound on the quantum capacity of a Pauli channel $23,32,9$. For a Pauli channel, this bound has a simple form and the proof that it is achievable is particularly simple as well. In this section, we summarize several variations of the hashing protocol for reliable communication in the asymptotic limit of many channel uses. In particular, one of the hashing bounds demonstrates that a maximal-entanglement EAQEC achieves the entanglement-assisted quantum capacity of a Pauli channel. We consider random stabilizer codes and use some techniques from the previous section. For more details about quantum Shannon theory, we refer interested readers to Ref. [43] and references therein.

\subsection{Hashing Bound for Stabilizer Codes}

We first review a simple proof of the hashing bound for stabilizer codes [35] in order to have it available for helping to obtain the proofs of the hashing bounds for EAQEC codes.

Theorem 10 (Hashing Bound) There exists a quantum stabilizer code that achieves the hashing limit $R=1-H(\mathbf{p})$ for a Pauli channel $\mathcal{E}$ of the following form:

$$
\mathcal{E}(\rho)=p_{I} \rho+p_{X} X \rho X+p_{Y} Y \rho Y+p_{Z} Z \rho Z,
$$

where $\rho$ is the density operator of a single qubit, $\mathbf{p}=\left(p_{I}, p_{X}, p_{Y}, p_{Z}\right)$ and $H(\mathbf{p})=-\sum_{i \in\{I, X, Y, Z\}} p_{i} \log _{2}\left(p_{i}\right)$ is the entropy of this probability vector.

Proof We need to correct only the typical errors. Define the typical error set as follows:

$$
T_{\delta}^{\mathbf{p}^{n}} \equiv\left\{a^{n}:\left|-\frac{1}{n} \log _{2}\left(\operatorname{Pr}\left\{E_{a^{n}}\right\}\right)-H(\mathbf{p})\right| \leq \delta\right\},
$$

where $a^{n}$ is some sequence consisting of the letters $\{I, X, Y, Z\}$ and $\operatorname{Pr}\left\{E_{a^{n}}\right\}$ is the probability that an independent and identically distributed (IID) Pauli channel issues some tensor-product error $E_{a^{n}} \equiv$ $E_{a_{1}} \otimes \cdots \otimes E_{a_{n}}$, where $E_{a_{j}} \in\{I, X, Y, Z\}$. This typical set consists of the likely errors in the sense that

$$
\sum_{a^{n} \in T_{\delta}^{\mathbf{p}^{n}}} \operatorname{Pr}\left\{E_{a^{n}}\right\} \geq 1-\epsilon
$$




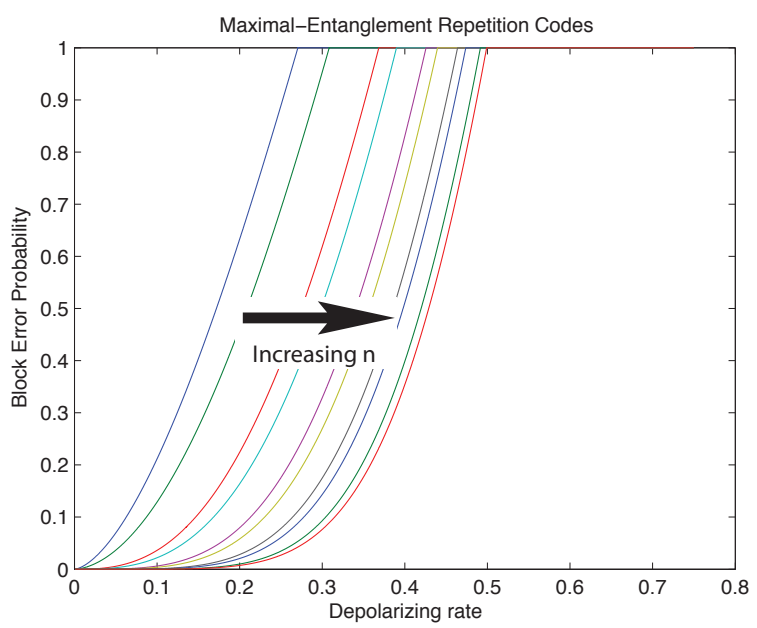

(a)

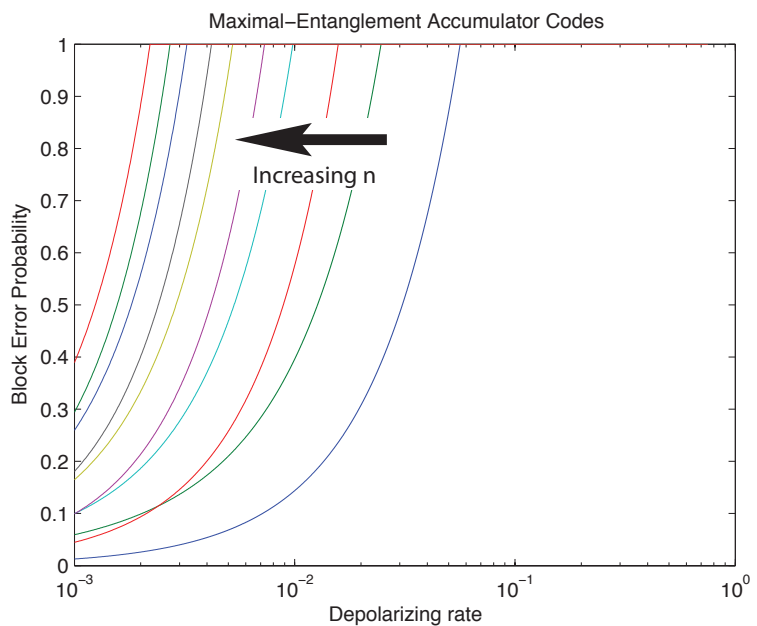

(c)

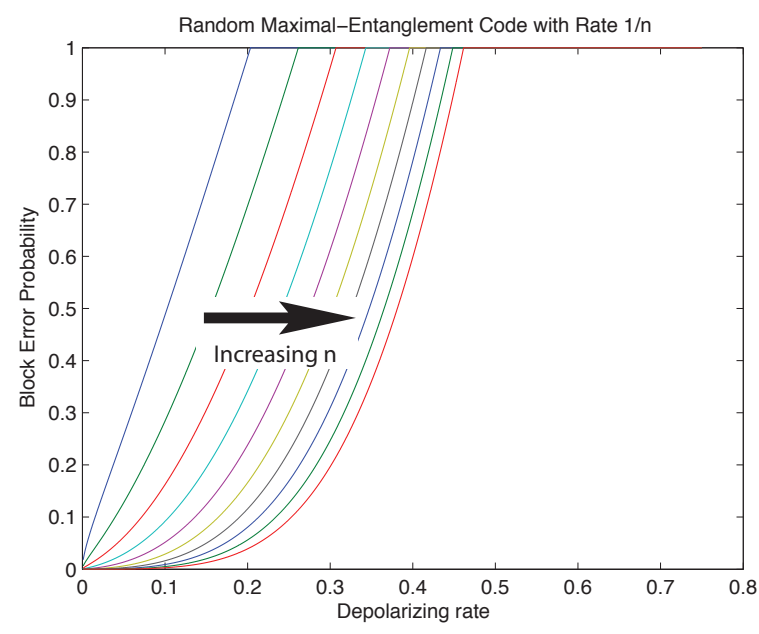

(b)

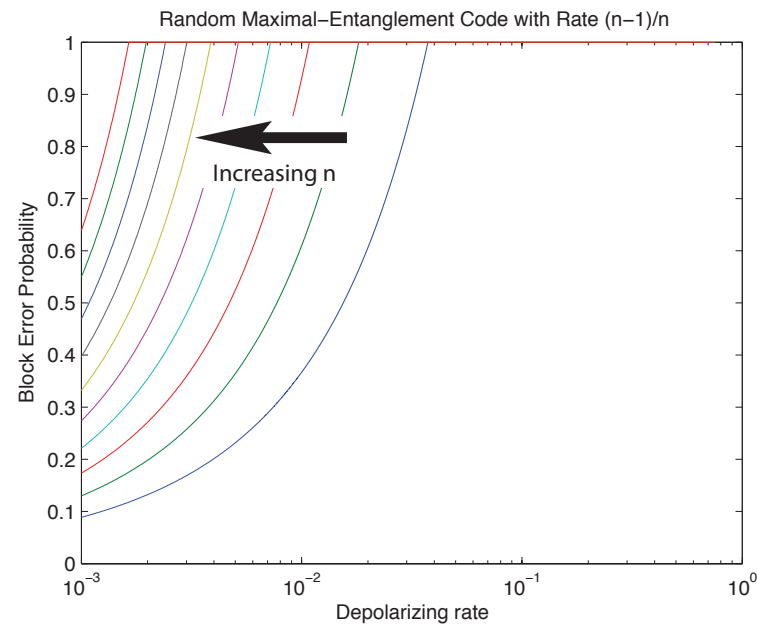

(d)

Fig. 3 The figures plot the weight enumerator bound in 14 as a function of the depolarizing parameter $p$ for various finite-length codes. (a) The weight enumerator bound for maximal-entanglement repetition codes of length 3 to 12 . (b) The expected weight enumerator bound for random rate $1 / n$ maximal-entanglement codes of length 3 to 12 . (c) The weight enumerator bound for maximal-entanglement accumulator codes of length 3 to 12. (d) The expected weight enumerator bound for random rate $(n-1) / n$ maximal-entanglement codes of length 3 to 12 . Observe that the performance of the maximal-entanglement repetition and accumulator codes with respect to this upper bound is comparable to the expected performance of random maximal-entanglement codes.

for all $\epsilon>0$ and sufficiently large $n$. The quantum error correction conditions for a stabilizer code defined by a stabilizer group $\mathcal{S}$ in this case are that $\left\{E_{a^{n}}: a^{n} \in T_{\delta}^{\mathrm{p}^{n}}\right\}$ is a correctable set of errors if

$$
E_{a^{n}}^{\dagger} E_{b^{n}} \notin N(\mathcal{S}) \backslash \tilde{\mathcal{S}}
$$

for all error pairs $E_{a^{n}}$ and $E_{b^{n}}$ such that $a^{n}, b^{n} \in T_{\delta}^{\mathbf{p}^{n}}$, where $\tilde{\mathcal{S}}=\{e g: g \in \mathcal{S}, e \in\{ \pm I, \pm i I\}\}$. Also, we consider the expectation of the error probability under a random choice of a stabilizer code and proceed 
to bound it as follows:

$$
\begin{aligned}
\mathbb{E}_{\mathcal{S}}\left\{p_{e}\right\} & =\mathbb{E}_{\mathcal{S}}\left\{\sum_{a^{n}} \operatorname{Pr}\left\{E_{a^{n}}\right\} \mathcal{I}\left(E_{a^{n}} \text { is uncorrectable under } \mathcal{S}\right)\right\} \\
& \leq \mathbb{E}_{\mathcal{S}}\left\{\sum_{a^{n} \in T_{\delta}^{\mathrm{p}^{n}}} \operatorname{Pr}\left\{E_{a^{n}}\right\} \mathcal{I}\left(E_{a^{n}} \text { is uncorrectable under } \mathcal{S}\right)\right\}+\epsilon \\
& =\sum_{a^{n} \in T_{\delta}^{\mathrm{p}^{n}}} \operatorname{Pr}\left\{E_{a^{n}}\right\} \mathbb{E}_{\mathcal{S}}\left\{\mathcal{I}\left(E_{a^{n}} \text { is uncorrectable under } \mathcal{S}\right)\right\}+\epsilon \\
& =\sum_{a^{n} \in T_{\delta}^{\mathrm{p}^{n}}} \operatorname{Pr}\left\{E_{a^{n}}\right\} \operatorname{Pr}_{\mathcal{S}}\left\{E_{a^{n}} \text { is uncorrectable under } \mathcal{S}\right\}+\epsilon .
\end{aligned}
$$

The first equality follows by definition: $\mathcal{I}$ is an indicator function equal to one if $E_{a^{n}}$ is uncorrectable under $\mathcal{S}$ and equal to zero otherwise. The first inequality follows from (16): we correct only the typical errors because the atypical error set has negligible probability mass. The second equality follows by exchanging the expectation and the sum. The third equality follows because the expectation of an indicator function is the probability that the event it selects occurs. Continuing, we have

$$
\begin{aligned}
& =\sum_{a^{n} \in T_{\delta}^{\mathbf{p}^{n}}} \operatorname{Pr}\left\{E_{a^{n}}\right\} \operatorname{Pr}_{\mathcal{S}}\left\{\exists E_{b^{n}}: b^{n} \in T_{\delta}^{\mathbf{p}^{n}}, b^{n} \neq a^{n}, E_{a^{n}}^{\dagger} E_{b^{n}} \in N(\mathcal{S}) \backslash \tilde{\mathcal{S}}\right\} \\
& \leq \sum_{a^{n} \in T_{\delta}^{A^{n}}} \operatorname{Pr}\left\{E_{a^{n}}\right\} \operatorname{Pr}_{\mathcal{S}}\left\{\exists E_{b^{n}}: b^{n} \in T_{\delta}^{\mathbf{p}^{n}}, b^{n} \neq a^{n}, E_{a^{n}}^{\dagger} E_{b^{n}} \in N(\mathcal{S})\right\} \\
& =\sum_{a^{n} \in T_{\delta}^{\mathbf{p}^{n}}} \operatorname{Pr}\left\{E_{a^{n}}\right\} \operatorname{Pr}_{\mathcal{S}}\left\{\bigcup_{b^{n} \in T_{\delta}^{\mathbf{p}^{n}}, b^{n} \neq a^{n}} E_{a^{n}}^{\dagger} E_{b^{n}} \in N(\mathcal{S})\right\} \\
& \leq \sum_{a^{n}, b^{n} \in T_{\delta}^{\mathbf{p}^{n}}, b^{n} \neq a^{n}} \operatorname{Pr}\left\{E_{a^{n}}\right\} \operatorname{Pr}_{\mathcal{S}}\left\{E_{a^{n}}^{\dagger} E_{b^{n}} \in N(\mathcal{S})\right\} \\
& \leq \sum_{a^{n}, b^{n} \in T_{\delta}^{\mathbf{p}^{n}}, b^{n} \neq a^{n}} \operatorname{Pr}\left\{E_{a^{n}}\right\} 2^{-(n-k)} \\
& \leq 2^{2 n[H(\mathbf{p})+\delta]} 2^{-n[H(\mathbf{p})+\delta]} 2^{-(n-k)} \\
& =2^{-n[1-H(\mathbf{p})-k / n-\delta]} .
\end{aligned}
$$

The first equality follows from the error correction conditions for a quantum stabilizer code. The first inequality follows by ignoring any potential degeneracy in the code - we consider an error uncorrectable if it lies in the normalizer $N(\mathcal{S})$ and the probability can only be larger because $N(\mathcal{S}) \backslash \tilde{\mathcal{S}} \subseteq N(\mathcal{S})$. The second equality follows by realizing that the probabilities for the existence criterion and the union of events are equivalent. The second inequality follows by applying the union bound. The third inequality follows from the fact that the probability for a fixed operator $E_{a^{n}}^{\dagger} E_{b^{n}}$ not equal to the identity commuting with the stabilizer operators of a random stabilizer can be upper bounded as follows:

$$
\operatorname{Pr}_{\mathcal{S}}\left\{E_{a^{n}}^{\dagger} E_{b^{n}} \in N(\mathcal{S})\right\}=\frac{2^{n+k}-1}{2^{2 n}-1} \leq 2^{-(n-k)} .
$$

The reasoning here is similar to the reasoning in Theorem 9. The random choice of a stabilizer code is equivalent to fixing operators $Z_{1}, \ldots, Z_{n-k}$ and performing a uniformly random Clifford unitary. The probability that a fixed operator commutes with $\bar{Z}_{1}, \ldots, \bar{Z}_{n-k}$ is then just the number of non-identity operators in the normalizer $\left(2^{n+k}-1\right)$ divided by the total number of non-identity operators $\left(2^{2 n}-1\right)$. After applying the above bound, we then exploit the following typicality bounds:

$$
\begin{aligned}
\forall a^{n} & \in T_{\delta}^{\mathbf{p}^{n}}: \operatorname{Pr}\left\{E_{a^{n}}\right\} \leq 2^{-n[H(\mathbf{p})+\delta]}, \\
\left|T_{\delta}^{\mathbf{p}^{n}}\right| & \leq 2^{n[H(\mathbf{p})+\delta]} .
\end{aligned}
$$


We conclude that as long as the rate $k / n=1-H(\mathbf{p})-2 \delta$, the expectation of the error probability becomes arbitrarily small, so that there exists at least one choice of a stabilizer code with the same bound on the error probability.

\subsection{Entanglement-Assisted Quantum Error-Correcting Codes}

\subsubsection{Maximal-Entanglement Codes}

Now consider the case of an EAQEC code. At first, we choose the code to be a maximal-entanglement EAQEC code, so that there are only information qubits or shares of ebits sent into the encoder. The quantum error correction conditions in such a case become that $\left\{E_{a^{n}}\right\}$ is a correctable set of errors if

$$
E_{a^{n}}^{\dagger} E_{b^{n}} \notin N\left(\mathcal{S}_{S}\right),
$$

for all error pairs $E_{a^{n}}$ and $E_{b^{n}}$ in the error set, where $\mathcal{S}_{S}$ is the symplectic subgroup of the stabilizer code. It follows for a random EAQEC code of this form that

$$
\operatorname{Pr}_{\mathcal{S}}\left\{E_{a^{n}}^{\dagger} E_{b^{n}} \in N\left(\mathcal{S}_{E}\right)\right\}=\frac{2^{2 k}-1}{2^{2 n}-1} \leq 2^{-2(n-k)}
$$

because there are $2^{2 k}-1$ nonidentity operators that commute with the $2(n-k)$ operators that generate $\mathcal{S}_{S}$. By modifying the last few steps of the above proof as follows

$$
\begin{aligned}
\sum_{a^{n}, b^{n} \in T_{\delta}^{\mathbf{p}^{n}}, b^{n} \neq a^{n}} \operatorname{Pr}\left\{E_{a^{n}}\right\} 2^{-2(n-k)} & \leq 2^{2 n[H(\mathbf{p})+\delta]} 2^{-n[H(\mathbf{p})+\delta]} 2^{-2(n-k)} \\
& =2^{-2 n[1-H(\mathbf{p}) / 2-k / n-\delta / 2]},
\end{aligned}
$$

we obtain the hashing bound for EAQEC codes:

Theorem 11 (EA Hashing Bound) There exists a maximal-entanglement EAQEC code that achieves the EA hashing limit $R=1-H(\mathbf{p}) / 2$ for a Pauli channel with parameters $\mathbf{p}$.

\subsubsection{Non-Maximal-Entanglement Codes}

We could also consider codes that do not use the maximal amount of ebits possible. In this case, there are $k$ information qubits, $n-k-c$ ancilla qubits, and $c$ ebits. The quantum error correction conditions in this case become that $\left\{E_{a^{n}}\right\}$ is a correctable set of errors if

$$
E_{a^{n}}^{\dagger} E_{b^{n}} \notin N\left(\mathcal{S}_{S}, \mathcal{S}_{I}\right) \backslash \tilde{\mathcal{S}}_{I}
$$

for all error pairs $E_{a^{n}}$ and $E_{b^{n}}$ in the error set, where $\mathcal{S}_{S}$ is the symplectic subgroup and $\mathcal{S}_{I}$ is the isotropic subgroup of the EAQEC code, and $\tilde{\mathcal{S}}_{I}=\left\{e g: g \in \mathcal{S}_{I}, e \in\{ \pm I, \pm i I\}\right\}$. Focusing only on non-denegerate errors, the error-correcting conditions become

$$
E_{a^{n}}^{\dagger} E_{b^{n}} \notin N\left(\mathcal{S}_{S}, \mathcal{S}_{I}\right)
$$

Then the relevant probability is

$$
\operatorname{Pr}_{\mathcal{S}}\left\{E_{a^{n}}^{\dagger} E_{b^{n}} \in N\left(\mathcal{S}_{S}, \mathcal{S}_{I}\right)\right\}=\frac{2^{n+k-c}-1}{2^{2 n}-1} \leq 2^{-(n-k+c)}=2^{-n(1-k / n+c / n)},
$$

which follows from similar counting arguments. This then leads to the following theorem for general EAQEC codes:

Theorem 12 (EA Hashing Region) There exists an EAQEC code whose achievable rate pair $(Q=k / n$, $E=c / n)$ obeys the following EA hashing bound for a Pauli channel with parameters $\mathbf{p}$ :

$$
Q \leq 1-H(\mathbf{p})+E .
$$

By varying $c$ from 0 to the maximal amount $n-k$, we can interpolate between stabilizer codes and maximal-entanglement EAQEC codes and achieve all rate pairs in the following hashing region:

$$
\begin{aligned}
& Q \leq 1-H(\mathbf{p})+E \\
& Q \leq 1-H(\mathbf{p}) / 2 .
\end{aligned}
$$




\subsubsection{Entanglement-Assisted Codes with Imperfect Ebits}

In the case that the ebits of the receiver are not perfect, we can use another stabilizer code to protect the ebits employed in the EAQEC code for transmitting information qubits 21,40. Suppose that Alice uses an $[[n, k ; c]]$ EAQEC code with a (simplified) stabilizer group $\mathcal{S}_{1}$ through a Pauli channel with parameter $\mathbf{p}_{1}$ to communicate with Bob and Bob's qubits suffer a Pauli channel with parameter $\mathbf{p}_{2}$. Furthermore, suppose Bob uses an $[[m, c]]$ stabilizer code with a stabilizer group $\mathcal{S}_{2}$ to protect his $c$ qubits.

Suppose Bob uses two decoders in sequence to correct the errors - the first corrects the errors on the ebits and the second corrects the errors on the information qubits. Following the proof of Theorem 10 and employing the union bound for two independent uses of the codes, we have the following hashing bound for combination codes when the ebits are imperfect:

Theorem 13 (Hashing Bounds for Combination Codes) Let $\alpha=\frac{m}{n}$. There exists an $[[n, k ; c]]$ $E A Q E C$ code combined with an $[[m, c]]$ stabilizer code with achievable rate pair $(Q=k / n, E=c / n)$ obeys the following hashing bounds for two Pauli channels with parameters $\mathbf{p}_{1}$ and $\mathbf{p}_{2}$, respectively:

$$
\begin{aligned}
\frac{1}{\alpha} E & \leq 1-H\left(\mathbf{p}_{2}\right), \\
Q & \leq 1-H\left(\mathbf{p}_{1}\right)+E .
\end{aligned}
$$

On the other hand, Bob can treat the combination code as an $[[n+m, k]]$ stabilizer code with a stabilizer group $\mathcal{S}$. Using a similar argument as in the proof of Theorem 10 we find that $Q \leq 1+\alpha-H\left(\mathbf{p}_{\mathbf{1}}\right)-\alpha H\left(\mathbf{p}_{\mathbf{2}}\right)$, which agrees with Theorem 13 if the entanglement consumption rate $E$ can be as large as $\alpha\left(1-H\left(\mathbf{p}_{\mathbf{2}}\right)\right)$. This result might be considered surprising because the simulations in Ref. 21] suggest that a single decoder has better performance than decoding the two codes in sequence -however, it appears that this is a finite blocklength effect that gets washed away in the asymptotic limit.

\subsection{EAQEC Codes for Classical Communication}

Now suppose the goal is to send classical data by exploiting maximal-entanglement EAQEC codes. In this case, the stabilizer structure is similar to that for a maximal-entanglement EAQEC code for sending quantum data, but this time we do not care if $Z$ errors affect the information qubits because they are classical. The error correction conditions then become that $\left\{E_{a^{n}}\right\}$ is a correctable set of errors if

$$
E_{a^{n}}^{\dagger} E_{b^{n}} \notin N\left(\mathcal{S}_{S}, \mathcal{L}_{X}\right),
$$

for all error pairs $E_{a^{n}}$ and $E_{b^{n}}$ in the error set, where $\mathcal{S}_{S}$ is the symplectic subgroup and $\mathcal{L}_{X}$ is the logical $X$ subgroup of the EAQEC code. Then the relevant probability is

$$
\operatorname{Pr}_{\mathcal{S}}\left\{E_{a^{n}}^{\dagger} E_{b^{n}} \in N\left(\mathcal{S}_{S}, \mathcal{L}_{X}\right)\right\}=\frac{2^{k}-1}{2^{2 n}-1} \leq 2^{-(2 n-k)}=2^{-n(2-k / n)},
$$

which follows from similar counting arguments. By modifying the last few steps of the proof of Theorem 10, we obtain the following upper bound:

$$
\begin{aligned}
\sum_{a^{n}, b^{n} \in T_{\delta}^{\mathbf{p}^{n}}, b^{n} \neq a^{n}} \operatorname{Pr}\left\{E_{a^{n}}\right\} 2^{-n(2-k / n)} & \leq 2^{2 n[H(\mathbf{p})+\delta]} 2^{-n[H(\mathbf{p})+\delta]} 2^{-n(2-k / n)} \\
& =2^{-n[2-H(\mathbf{p})-k / n-\delta]},
\end{aligned}
$$

giving the EA hashing bound for classical communication:

Theorem 14 (EA Hashing Bound for Classical Communication) There exists an EAQEC code for classical communication that achieves the EA classical hashing limit $R=2-H(\mathbf{p})$ for a Pauli channel with parameters $\mathbf{p}$. 


\section{Discussion}

In this paper, we studied several properties of EAQEC codes, including the duality of EAQEC codes, the MacWilliams identities for EAQEC codes, and the linear programming bound on the minimum distance of an EAQEC code. We also derived the Plotkin bound and the Gilbert-Varshamov bound for EAQEC codes, together with several theorems examining the existence of EAQEC codes. Finally, we determined "weight enumerator bounds" on the block error probability when decoding according to a maximumlikelihood decoding rule, and we found that the performance of maximal-entanglement repetition and accumulator codes is comparable to the expected performance of random codes, with respect to this upper bound.

The table of upper and lower bounds on the minimum distance of any $[[n, k, d]]$ standard stabilizer codes $(c=0)$ is given in [7. Similar tables for EAQEC codes with $0<c<n-k$ can be constructed.

We proposed a construction of $[[n, 1, n-1 ; n-1]]$ EA repetition codes for $n$ even, which completes the family of EA repetition codes for any $n$. These EA repetition codes are the optimal codes that encode a single information qubit. We also constructed an explicit encoding circuit for these codes. We also proved the non-existence of $[[n, 1, n ; n-1]]$ or $[[n, n-1,2 ; 1]]$ codes for $n$ even, which decreases the upper bound predicted by the linear programming bound for $k=1$ and $n$ even.

We plan to explore the existence of other $[[n, k, d ; n-k]]$ codes to decrease the upper bound. Consider the possibility of a "self-dual code" $[[n, n / 2, d ; n / 2]]$ for $n$ even, such that the dual code is also an $[[n, n / 2, d ; n / 2]]$ code with the same weight enumerators. That is, $W_{\mathcal{L}}(x, y)=W_{\mathcal{S}_{S}}(x, y)$. We conjecture that such self-dual codes exist. If so, the two groups $\mathcal{S}_{S}$ and $\mathcal{L}$ may be equivalent up to a permutation on the qubits. Such codes would have interesting and useful properties.

Finally, we applied the idea of random stabilizer codes to prove an upper bound on the average block error rate, and we also proved several variations of the hashing bound for EAQEC codes. It should be possible to improve upon the hashing regions by exploiting degeneracy in EAQEC codes, by an approach similar to that from Ref. [36].

TAB and CYL were supported in part by NSF Grant CCF-0830801. This work was supported in part by the Intelligence Advanced Research Projects Activity (IARPA) via Department of Interior National Business Center contract number D11PC20165. The U.S. Government is authorized to reproduce and distribute reprints for Governmental purposes notwithstanding any copyright annotation thereon. The views and conclusions contained herein are those of the authors and should not be interpreted as necessarily representing the official policies or endorsements, either expressed or implied, of IARPA, DoI/NBC or the U.S. Government. MMW acknowledges the support of the MDEIE (Québec) PSR-SIIRI international collaboration grant. MMW acknowledges useful discussions with Omar Fawzi and Jan Florjanczyk.

\section{References}

1. Ashikhmin, A., Litsyn, S.: Upper bounds on the size of quantum codes. IEEE Trans. Inform. Theory 45(4), 1206 1215 (1999)

2. Bennett, C.H., DiVincenzo, D.P., Smolin, J.A., Wootters, W.K.: Mixed state entanglement and quantum error correction. Phys. Rev. A 54(5), 3824-3851 (1996). URL http://arxiv.org/abs/quant-ph/9604024

3. Bennett, C.H., Shor, P.W., Smolin, J.A., Thapliyal, A.V.: Entanglement-assisted classical capacity of noisy quantum channels. Phys. Rev. Lett. 83(15), 3081-3084 (1999). DOI 10.1103/PhysRevLett.83.3081

4. Bowen, G.: Entanglement required in achieving entanglement-assisted channel capacities. Phys. Rev. A 66, 052,313 (2002)

5. Brun, T.A., Devetak, I., Hsieh, M.-H.: Correcting quantum errors with entanglement. Science 314, 436-439 (2006)

6. Calderbank, A.R., Rains, E.M., Shor, P.W., Sloane, N.J.A.: Quantum error correction and orthogonal geometry. Phys. Rev. Lett. 78(3), 405-408 (1997). URL http://arxiv.org/abs/quant-ph/9605005

7. Calderbank, A.R., Rains, E.M., Shor, P.W., Sloane, N.J.A.: Quantum error correction via codes over GF(4). IEEE Trans. Inform. Theory 44(4), 1369-1387 (1998). URL http://arxiv.org/abs/quant-ph/9608006

8. Calderbank, A.R., Shor, P.W.: Good quantum error-correcting codes exist. Phys. Rev. A 54(2), 1098-1105 (1996). URL http://arxiv.org/abs/quant-ph/9512032

9. Devetak, I.: The private classical capacity and quantum capacity of a quantum channel. IEEE Trans. Inform. Theory 51(1), 44-55 (2005). DOI 10.1109/TIT.2004.839515

10. Devetak, I., Harrow, A.W., Winter, A.: A family of quantum protocols. Phys. Rev. Lett. 93(23), 230,504 (2004). DOI 10.1103/PhysRevLett.93.230504

11. Devetak, I., Harrow, A.W., Winter, A.: A resource framework for quantum Shannon theory. IEEE Trans. Inform. Theory 54(10), 4587-4618 (2008)

12. DiVincenzo, D.P., Leung, D.W., Terhal, B.M.: Quantum data hiding. IEEE Trans. Inform. Theory 48(3), 580-598 (2002) 
13. Ekert, A., Macchiavello, C.: Quantum error-correction for communication. Phys. Rev. Lett. 77(12), 2585-2588 (1996)

14. Gottesman, D.: Stabilizer codes and quantum error correction. Ph.D. thesis, California Institute of Technology, Pasadena, CA (1997). URL http://arxiv.org/abs/quant-ph/9705052

15. Guo, L., Li, R.: Linear plotkin bound for entanglement-assisted quantum codes. Phys. Rev. A 87, 032,309 (2013). DOI 10.1103/PhysRevA.87.032309. URL http://link.aps.org/doi/10.1103/PhysRevA.87.032309

16. Hsieh, M.-H., Brun, T.A., Devetak, I.: Entanglement-assisted quantum quasi-cyclic low-density parity-check codes. Phys. Rev. A 79, 032,340 (2009). URL http://arxiv.org/abs/0803.0100

17. Hsieh, M.-H., Yen, W.-T., Hsu, L.-Y.: High performance entanglement-assisted quantum ldpc codes need little entanglement. IEEE Trans. Inform. Theory 57(3), 1761-1769 (2011). DOI 10.1109/TIT.2011.2104590

18. Knapp, A.W.: Basic Algebra. Boston:Birkhäuser (2006)

19. Knill, E., Laflamme, R.: A theory of quantum error-correcting codes. Phys. Rev. A 55(2), 900-911 (1997)

20. Lai, C.-Y., Brun, T.A.: Entanglement increases the error-correcting ability of quantum error-correcting codes (2010). URL http://arxiv.org/abs/1008.2598v1

21. Lai, C.-Y., Brun, T.A.: Entanglement-assisted quantum error-correcting codes with imperfect ebits. Phys. Rev. A 86, 032,319 (2012). DOI 10.1103/PhysRevA.86.032319. URL http://link.aps.org/doi/10.1103/PhysRevA.86.032319

22. Lai, C.-Y., Brun, T.A., Wilde, M.M.: Duality in entanglement-assisted quantum error correction. IEEE Trans. Inform. Theory 59(6), 4020-4024 (2013). DOI 10.1109/TIT.2013.2246274.

23. Lloyd, S.: Capacity of the noisy quantum channel. Phys. Rev. A 55, 1613-1622 (1997). DOI 10.1103/PhysRevA.55.1613. URL http://link.aps.org/doi/10.1103/PhysRevA.55.1613

24. MacWilliams, F.J., Sloane, N.J.A.: The Theory of Error-Correcting Codes. North-Holland, Amsterdam, The Netherlands (1977)

25. McEliece, R.J.: The Theory of Information and Coding. Cambridge University Press (2002)

26. Nielsen, M.A., Chuang, I.L.: Quantum Computation and Quantum Information. Cambridge University Press, Cambridge, UK (2000)

27. Poulin, D., Tillich, J.P., Ollivier, H.: Quantum serial turbo codes. IEEE Trans. Inform. Theory 55(6), 2776-2798 (2009). DOI http://dx.doi.org/10.1109/TIT.2009.2018339

28. Rains, E.M.: Quantum weight enumerators. IEEE Trans. Inform. Theory 44(4), 1388 - 1394 (1995)

29. Rains, E.M.: Monotonicity of the quantum linear programming bound. IEEE Trans. Inform. Theory 45(7), 2489 $2492(1999)$

30. Richardson, T., Urbanke, R.: Modern Coding Theory. Cambridge University Press (2008)

31. Shaw, B., Wilde, M.M., Oreshkov, O., Kremsky, I., Lidar, D.A.: Encoding one logic qubit into six physical qubits. Phys. Rev. A 78, 012,337 (2008)

32. Shor, P.: The quantum channel capacity and coherent information. In: Lecture Notes, MSRI Workshop on Quantum Computation (2002)

33. Shor, P., Laflamme, R.: Quantum analog of the MacWilliams identities for classical coding theory. Phys. Rev. Lett. 78(8), 1600-1602 (1997). DOI 10.1103/PhysRevLett.78.1600

34. Shor, P.W.: Scheme for reducing decoherence in quantum computer memory. Phys. Rev. A 52(4), 2493-2496 (1995)

35. Smith, G.: Upper and lower bounds on quantum codes. Ph.D. thesis, California Institute of Technology, Pasadena, CA (2006)

36. Smith, G., Smolin, J.A.: Degenerate quantum codes for pauli channels. Phys. Rev. Lett. 98, 030,501 (2007). DOI 10.1103/PhysRevLett.98.030501. URL http://link.aps.org/doi/10.1103/PhysRevLett.98.030501

37. Steane, A.M.: Error correcting codes in quantum theory. Phys. Rev. Lett. 77(5), $793-797$ (1996)

38. Steane, A.M.: Multiple particle interference and quantum error correction. Proc. R. Soc. London A 452, 2551-2576 (1996). URL http://arxiv.org/abs/quant-ph/9601029

39. Steane, A.M.: Simple quantum error-correcting codes. Phys. Rev. A 54(6), 4741-4751 (1996)

40. Wilde, M.M., Hsieh, M.-H.: Entanglement boosts quantum turbo codes. In: Information Theory Proceedings (ISIT), 2011 IEEE International Symposium on, pp. 445-449 (31 2011-Aug. 5). DOI 10.1109/ISIT.2011.6034165

41. Wilde, M.M.: Quantum coding with entanglement. Ph.D. thesis, University of Southern California (2008). URL http://arxiv.org/abs/0806.4214

42. Wilde, M.M.: Quantum-shift-register circuits. Phys. Rev. A 79(6), 062,325 (2009). DOI 10.1103/PhysRevA.79.062325

43. Wilde, M.M.: From Classical to Quantum Shannon Theory. URL http://arxiv.org/abs/1106.1445

44. Wilde, M.M., Brun, T.A.: Optimal entanglement formulas for entanglement-assisted quantum coding. Phys. Rev. A 77, 064,302 (2008). URL doi:10.1103/PhysRevA.77.064302

45. Wilde, M.M., Brun, T.A.: Entanglement-assisted quantum convolutional coding. Phys. Rev. A 81, 042,333 (2010). DOI 10.1103/PhysRevA.81.042333. URL http://link.aps.org/doi/10.1103/PhysRevA.81.042333 